\title{
An Eulerian projection method for quasi-static elastoplasticity
}

\author{
Chris H. Rycroft ${ }^{\mathrm{a}, \mathrm{b}, *}$, Yi Sui ${ }^{\mathrm{c}}$, Eran Bouchbinder ${ }^{\mathrm{d}}$ \\ ${ }^{a}$ School of Engineering and Applied Sciences, Harvard University, Cambridge, MA 02138, United States \\ ${ }^{b}$ Department of Mathematics, Lawrence Berkeley Laboratory, Berkeley, CA 94720, United States \\ ${ }^{c}$ Mathematics Department, University of British Columbia, Vancouver, British Columbia, V6T 1Z4, Canada \\ ${ }^{d}$ Chemical Physics Department, Weizmann Institute of Science, Rehovot 76100, Israel
}

\begin{abstract}
A well-established numerical approach to solve the Navier-Stokes equations for incompressible fluids is Chorin's projection method [1], whereby the fluid velocity is explicitly updated, and then an elliptic problem for the pressure is solved, which is used to orthogonally project the velocity field to maintain the incompressibility constraint. In this paper, we develop a mathematical correspondence between Newtonian fluids in the incompressible limit and hypo-elastoplastic solids in the slow, quasi-static limit. Using this correspondence, we formulate a new fixed-grid, Eulerian numerical method for simulating quasi-static hypo-elastoplastic solids, whereby the stress is explicitly updated, and then an elliptic problem for the velocity is solved, which is used to orthogonally project the stress to maintain the quasi-staticity constraint. We develop a finite-difference implementation of the method and apply it to an elasto-viscoplastic model of a bulk metallic glass based on the shear transformation zone theory. We show that in a two-dimensional plane strain simple shear simulation, the method is in quantitative agreement with an explicit method. Like the fluid projection method, it is efficient and numerically robust, making it practical for a wide variety of applications. We also demonstrate that the method can be extended to simulate objects with evolving boundaries. We highlight a number of correspondences between incompressible fluid mechanics and quasi-static elastoplasticity, creating possibilities for translating other numerical methods between the two classes of physical problems.
\end{abstract}

Keywords: fluid mechanics, projection method, plasticity, elastoplasticity

\section{Introduction}

A wide variety of materials of scientific and technological importance exhibit elastoplastic behavior, such as metals [2,3], granular materials [4], aerogels [5], and amorphous solids such as bulk metallic glasses (BMGs) [6]. At low levels of stress these materials typically behave elastically, so that the deformation they undergo is reversible when the stress is removed. However, at higher levels of stress, the material will start to yield, and undergo plastic, irreversible deformation that will remain

\footnotetext{
${ }^{*}$ Corresponding author.

Email addresses: chr@seas . harvard . edu (Chris H. Rycroft), eran. bouchbinder@weizmann . ac. il (Eran Bouchbinder)
} 
after the stress is removed. Describing elastoplastic ${ }^{1}$ behavior within a consistent theoretical framework has been the subject of major research effort over many decades, particularly from the 1950's onward. As described in a recent review article [7], accurately characterizing elastoplastic behavior has proved challenging, since it is not obvious how to separate the elastic and plastic response at the microscopic level. Several different frameworks have emerged, each of which is based on different assumptions of how the elastic and plastic behavior are combined.

Currently, perhaps the most widely-used framework to study elastoplastic materials is hyperelastoplasticity $[8,9]$. This model is based on introducing a initial undeformed reference configuration of a material. A time-dependent mapping is then employed, transforming the reference configuration into the deformed configuration at a later time. The deformation gradient tensor $\mathbf{F}$ is then defined as the Jacobian matrix of the mapping, and represents how an infinitesimal material element is transformed. A purely elastic material can then be described in terms of a constitutive law that gives stress as a function of $\mathbf{F}$. To generalize this to elastoplastic behavior, the Kröner-Lee decomposition was developed, whereby the deformation gradient tensor is viewed as the product of elastic and plastic parts, $\mathbf{F}=\mathbf{F}_{e} \mathbf{F}_{p}[10,11]$. This decomposition has been successfully used to model the elastoplastic behavior of a variety of materials such as metals and metallic glasses [12,13,14], and can be carried out in commercial solid mechanics software such as ABAQUS. However, the decomposition has also been extensively debated within the literature. For materials that undergo very large plastic deformation and rearrangement, the notion of a mapping from an initial configuration may become problematic. The decomposition is non-unique, whereby the stress remains invariant under the transformation of the intermediate configuration $\left(\mathbf{F}_{e}, \mathbf{F}_{p}\right) \mapsto\left(\mathbf{F}_{e} \mathbf{R}^{\top}, \mathbf{R F}_{p}\right)$ for an arbitrary rotation R. While $\mathbf{F}_{e}$ and $\mathbf{F}_{p}$ remain useful mathematical quantities, they may no longer retain their expected physical interpretations [7], which has led to recent efforts to clarify this from a micromechanical perspective, at least for crystalline solids [15].

An alternative framework is hypo-elastoplasticity, which is based on an additive decomposition of the Eulerian rate-of-deformation tensor into elastic and plastic parts, $\mathbf{D}=\mathbf{D}^{\mathrm{el}}+\mathbf{D}^{\mathrm{pl}}[16,17,18]$. This approach has some drawbacks: it has mainly been applied to elastoplastic simulations involving only linear elastic deformation, since it is difficult to capture a nonlinear elastic strain response purely through $\mathbf{D}^{\mathrm{el}}$. In particular, several researchers have noted some undesirable effects of the decomposition [19,20], such as leaving a residual stress after an elastic strain cycle [21]. Furthermore, because the framework is based on velocity as opposed to deformation, it can lead to the build-up of numerical errors during time-integration [22, 23]. However, because it is based on Eulerian quantities, it does not depend on an undeformed configuration, which is a potential advantage for materials undergoing large strains. The aforementioned difficulties are typically minor in the limit of small elastic deformation, and hence it may provide a reasonable framework for many materials such as metals and metallic glasses that have large elastic constants.

Another feature of hypo-elastoplasticity is that it naturally fits within an Eulerian, fixed-grid framework, and there are several recent trends in numerical computation that make fixed-grid

\footnotetext{
${ }^{1}$ Throughout this article, we use "elastoplastic" to refer to any material response that is a combination of reversible elastic deformation and irreversible plastic deformation. This includes, for example, rate-independent elastic-perfectly plastic models and rate-dependent elasto-viscoplastic models.
} 
methods desirable. A fixed grid has simpler topology, making it easier and more efficient to program, and simpler to parallelize. Eulerian methods are also a natural environment in which fluid-structure interactions are accounted for, since fixed-grid frameworks are often the technique of choice for fluids [24, 25]. Several approaches for dealing with nonlinear hyperelasticity have been proposed by treating the deformation gradient tensor as an Eulerian field [26, 27, 28] or by introducing a reference map field that describes the deformation from the initial undeformed state $[29,30,31,32]$. Other physical effects such as coupling to electrical fields [33] or the diffusion of temperature fit well within an Eulerian framework. Some manufacturing processes featuring continuous motion of material, such as extrusion [34], are also well-suited to the Eulerian viewpoint.

Starting from the additive decomposition of $\mathbf{D}$, and coupling it with a continuum version of Newton's second law, one ends up with a closed system of partial differential equations for velocity, stress, and typically a set of additional internal variables. From this system a direct, explicit numerical scheme can be constructed. The scheme resolves elastic waves in the material, leading to a restriction on the numerical timestep due to the Courant-Friedrichs-Lewy (CFL) condition. For many materials of interest, such as metals, the elastic wave speed is on the order of kilometers per second, which makes it prohibitive to simulate processes on physically relevant time scales of seconds, hours, or days. Because of this, most applications of hypo-elastoplasticity have been interested in rapid processes such as impact [35], or have scaled the elastic constants to be artificially soft [36]. If one scales the hypo-elastoplasticity equations to examine the long timescale and small velocity limit, one finds that the continuum version of Newton's second law can be replaced with a constraint that the stresses remain in quasi-static equilibrium.

In this paper, we show that there is a strong mathematical connection between quasi-static hypoelastoplasticity and the incompressible Navier-Stokes equations. For an incompressible fluid, the relevant variables are the velocity and pressure. There is an explicit update equation for velocity, and the incompressibility constraint requires that the velocity remain divergence-free. In this situation, a well-established method of solution is the projection method of Chorin [1], described in detail in Subsec. 2.2, whereby the fluid velocity is explicitly updated, and then an elliptic problem for the pressure is solved, which is used to orthogonally project the velocity field to maintain the incompressibility constraint. By exploiting the mathematical correspondence, we have developed a new numerical method for quasi-static elastoplasticity that is analogous to the projection method for incompressible fluid dynamics. It takes an analogous approach, whereby the stress is explicitly updated, and then an elliptic problem for the velocity is solved, which is used to orthogonally project the stress to maintain the quasi-staticity constraint.

To the best of our knowledge, this mathematical correspondence has not been noted and explored in detail before, and the resultant numerical method based on a projection step to restore quasi-staticity is distinct from existing computational approaches. Some of the most well-established numerical methods make use of an updated Lagrangian formulation and a mesh that deforms with the material [37, 38, 39]. Ponthot [40] developed an implicit simulation approach for elastoplasticity, although it again makes use of a moving-mesh framework, leading to different mathematical considerations. A number of authors developed and analyzed two-step algorithms for rate-independent plasticity, which involve an elastic predictor step followed by a plastic corrector step whereby the stress is projected to the yield surface $[41,42,43,44,45,46]$. However, this notion of a projection, 
which is carried out for each material element, is distinctly different from the global stress projection that we develop here.

In Section 2, we describe the mathematical correspondence to incompressible fluid mechanics and the associated numerical procedure. In Section 3, to illustrate the method, we develop a finitedifference implementation of it to study a specific rate-dependent, elasto-viscoplastic model of a bulk metallic glass based on the shear transformation zone (STZ) theory. Originally developed by Falk and Langer [47], this model has undergone significant development [48, 49], and has been applied to a wide variety of amorphous materials. The STZ model of the bulk metallic glass is an appropriate numerical example, since BMGs can undergo large amounts of plastic deformation in certain situations (such as at high temperature), and have elastic moduli on the order of 10-100 GPa, meaning that experimental tests are often in the quasi-static regime. A previous study that examined cavitation as a fracture mechanism in the STZ model specifically described the long timescale limit and made use of the quasi-staticity constraint for theoretical analysis [50].

While our numerical examples focus on the STZ model of a BMG, we note that the core of the numerical approach can be applied to a wide variety of plasticity models and physical problems. It could apply to other descriptions of BMGs, such as free-volume-based models $[51,52,53]$, which result in equations with a similar mathematical structure. It could also be applied to hypo-elastic materials or to rate-independent plasticity models. The method is not limited to the finite-difference method, and alternative discretization procedures could be used, such as the finite-volume or discontinuous Galerkin methods.

The first numerical example we present is a BMG undergoing simple shear deformation in a two-dimensional, plane strain, periodic geometry, which is simple enough to allow for quantitative analysis (Section 4). By choosing parameters appropriately, we quantitatively compare the quasi-static projection method to the explicit scheme. We provide numerical evidence that the two methods agree in the quasi-static limit. We also show that the quasi-static method can simulate elastoplastic dynamics on physically realistic timescales.

Many important problems of interest involve moving boundaries and hence we need an Eulerian description of such evolving boundaries. In Section 5 we extend the method to implement a tractionfree boundary condition at a boundary described by the level set method $[54,55,56]$. Finally, since the projection method makes use of the same numerical framework as the explicit scheme, the two methods can be interchanged making it possible to simulate phenomena on multiple disparate timescales. We previously demonstrated this capability to examine dynamic crack propagation [57]. Here, we present another case, of a bar that is loaded on a slow, quasi-static timescale and then released, undergoing rapid vibrations.

While many computational methods for elastoplasticity are already available, we find that the numerical method developed here offers a useful practical approach for dealing with hypo-elastoplastic materials in the quasi-static limit. One of the main advantages of the fluid projection method is that it maintains the incompressibility condition through a single algebraic problem for the pressure, which is generally well-conditioned and can be carried out efficiently, and we find that many of the same benefits remain valid for the elasto-plasticity method we develop. Throughout the paper, we find a surprising number of correspondences between the two methods, such as analogous considerations for boundary conditions or the uniqueness of solutions. The mathematical connection opens up 
interesting possibilities for translating numerical methods for incompressible fluid mechanics over to quasi-static elastoplasticity and vice versa.

\section{Theoretical development}

\subsection{An elastoplastic material model}

We consider an elastoplastic material with velocity $\mathbf{v}(\mathbf{x}, t)$ and Cauchy stress tensor $\boldsymbol{\sigma}(\mathbf{x}, t)$. The spin is defined as $\boldsymbol{\omega}=\nabla \times \mathbf{v}$, and the rate-of-deformation tensor is $\mathbf{D}=\left(\nabla \mathbf{v}+(\nabla \mathbf{v})^{\top}\right) / 2$. For an arbitrary field $f(\mathbf{x}, t)$, we define the advective derivative as $d f / d t=\partial f / \partial t+(\mathbf{v} \cdot \nabla) f$. Using the hypo-elastoplastic kinematic relation, the rate-of-deformation tensor is assumed to be the sum of elastic and plastic parts such that $\mathbf{D}=\mathbf{D}^{\mathrm{el}}+\mathbf{D}^{\mathrm{pl}}$. The linear elastic constitutive relation is

$$
\frac{\mathcal{D} \boldsymbol{\sigma}}{\mathcal{D} t}=\mathbf{C}: \mathbf{D}^{\mathrm{el}}=\mathbf{C}:\left(\mathbf{D}-\mathbf{D}^{\mathrm{pl}}\right)
$$

where $\mathbf{C}$ is a fourth-rank stiffness tensor, which for simplicity of presentation is assumed to be isotropic, and constant in space and time. The left hand side of Eq. 1 is the Jaumann objective stress rate, $\mathcal{D} \boldsymbol{\sigma} / \mathcal{D} t=d \boldsymbol{\sigma} / d t+\boldsymbol{\sigma} \cdot \boldsymbol{\omega}-\boldsymbol{\omega} \cdot \boldsymbol{\sigma}$, which gives the time-evolution of the stress taking into account translation and rotation of the material, under the assumption that the elastic deformation is small [58]. By considering force balance, the velocity satisfies

$$
\rho \frac{d \mathbf{v}}{d t}=\nabla \cdot \boldsymbol{\sigma}
$$

where $\rho$ is the density of the material. Taken together, Eqs. 1 and 2 form a hyperbolic system of equations from which a finite-difference simulation of an elastoplastic material can be constructed. However, the hyperbolic system will resolve the propagation of elastic waves, and therefore the timestep $\Delta t$ and grid spacing $\Delta x$ must be chosen to satisfy the CFL condition for numerical stability to be maintained. If $c_{e}$ is an elastic wave speed, then the timestep must satisfy $\Delta t \leq \Delta x / c_{e}$. For many problems of practical importance, such as simulating metals, this will pose a prohibitively strong restriction. A typical elastic wave speed would be on the order of kilometers per second, while a grid spacing could be on the order of millimeters to micrometers, thus requiring a timestep on the order of microseconds or smaller. This restriction would make it infeasible to simulate real problems on the timescale of seconds, minutes, or hours.

We now consider the limit when the deformation of the material happens on a time scale that is much longer than the time for elastic waves to propagate across the system. We rescale the equations in the limit of long times and corresponding small velocity gradients by introducing

$$
\nabla \mathbf{v}=\varepsilon \widetilde{\nabla \mathbf{v}}, \quad t=\frac{\tilde{t}}{\varepsilon}
$$

where $\varepsilon$ is a small dimensionless parameter. Under these scalings, the constitutive equation becomes

$$
\frac{\mathcal{D} \boldsymbol{\sigma}}{\mathcal{D} \tilde{t}}=\mathbf{C}:\left(\tilde{\mathbf{D}}-\frac{\mathbf{D}^{\mathrm{pl}}}{\varepsilon}\right),
$$


where $\tilde{\mathbf{D}}=\left(\widetilde{\nabla \mathbf{v}}+(\widetilde{\nabla \mathbf{v}})^{\top}\right) / 2$, and the force balance equation becomes

$$
\varepsilon \rho \frac{d \mathbf{v}}{d \tilde{t}}=\nabla \cdot \boldsymbol{\sigma}
$$

There are two occurrences of $\varepsilon$ in these equations. The $\varepsilon^{-1}$ in Eq. 4 signifies that over long durations, plastic deformation will become increasingly important, while the $\varepsilon$ term on $d \mathbf{v} / d t$ signifies that accelerations decrease in importance. Through these considerations, one can approximate the material response by neglecting the $d \mathbf{v} / d \tilde{t}$ term to give

$$
\nabla \cdot \boldsymbol{\sigma}=\mathbf{0}
$$

which physically states that forces remain in quasi-static equilibrium. A numerical scheme could then be constructed using the constitutive equation Eq. 1 subject to the constraint in Eq. 6. However, this would raise several questions. It is not clear how to update the velocity, since the ability to explicitly time-integrate it is lost. It is also not clear whether solutions of this system will match the solutions of the original system.

\subsection{Review of the projection method for the incompressible Navier-Stokes equations}

To make progress with the above problem, we now consider a different class of problems involving an incompressible fluid with velocity $\mathbf{v}$, pressure $p$, and density $\rho$. The fluid velocity satisfies the Navier-Stokes equations,

$$
\rho \frac{d \mathbf{v}}{d t}=-\nabla p+\nabla \cdot \mathbf{T}
$$

where $\mathbf{T}$ is the fluid stress tensor, and the fluid density will evolve according to

$$
\frac{d \rho}{d t}=-\rho(\nabla \cdot \mathbf{v})
$$

In addition, an equation of state linking the fluid density to the pressure must be satisfied. For typical weakly compressible fluids, the equation $\rho-\rho_{0}=\left(p-p_{0}\right) / c^{2}$ is appropriate, where $\rho_{0}$ and $p_{0}$ are reference densities and pressures respectively, and $c$ is a large constant that corresponds to a sound wave speed through the fluid.

In a similar manner to the elastoplastic system of equations considered in the previous section, Eqs. 7 and 8 form a hyperbolic system of equations that could be used to construct an explicit finite-difference simulation of the fluid, but due to the CFL condition, the presence of the sound speed places a severe restriction on the timestep size. Again, for many practical problems, one may wish to consider time scales that are much longer than the time for compressive waves to propagate across the system. Looking at long times by introducing $t=\varepsilon \tilde{t}$ as in Eq. 3, one finds that

$$
\varepsilon \frac{d \rho}{d \tilde{t}}=-\rho(\nabla \cdot \mathbf{v})
$$

which can be approximated by

$$
\nabla \cdot \mathbf{v}=0
$$


so that the velocity is divergence-free. The resultant system given by Eqs. 7 and 10 are the incompressible Navier-Stokes equations.

Numerical methods to simulate the incompressible Navier-Stokes equations have been extensively studied and developed. In work by Chorin [59], aiming at addressing the constraint imposed by Eq. 10, the incompressible Navier-Stokes equations were simulated by examining the compressible system as the parameter $c$ becomes large. Numerical evidence shows that in the limit in which $c$ becomes large, the compressible solutions approach the incompressible ones. This can also be understood by introducing a vector space $V_{\mathbf{v}}$ of all velocity fields. The divergence-free solutions $\mathbf{v} \in V_{\mathbf{v}}$, which satisfy $\nabla \cdot \mathbf{v}=0$, form a subspace in $V_{\mathbf{v}}$. In the compressible case, the $d \rho / d t$ term in Eq. 9, in tandem with the pressure gradient in Eq. 7, force the system toward being divergence-free.

This observation can be used as the basis of the projection method for incompressible NavierStokes equations [1]. Suppose that $\mathbf{v}_{n}$ represents the discretized velocity field after $n$ steps in a finite-difference simulation. To advance forward by $\Delta t$ to the $(n+1)$ th step an intermediate velocity $\mathbf{v}_{\star}$ is first computed by neglecting the pressure term, so that

$$
\frac{\rho\left(\mathbf{v}_{*}-\mathbf{v}_{n}\right)}{\Delta t}=-\left(\mathbf{v}_{n} \cdot \nabla\right) \mathbf{v}_{n}+\nabla \cdot \mathbf{T}_{n}
$$

If the pressure at the $(n+1)$ th step was known then $\mathbf{v}_{n+1}$ could be computed according to

$$
\frac{\mathbf{v}_{n+1}-\mathbf{v}_{*}}{\Delta t}=-\frac{1}{\rho} \nabla p_{n+1}
$$

Taking the divergence of Eq. 12 and enforcing that $\nabla \cdot \mathbf{v}_{n+1}=0$ gives

$$
\nabla \cdot \mathbf{v}_{*}=\frac{\Delta t}{\rho} \nabla \cdot\left(\nabla p_{n+1}\right)=\frac{\Delta t}{\rho} \nabla^{2} p_{n+1}
$$

and hence the pressure satisfies a Poisson equation where the source term is $\nabla \cdot \mathbf{v}_{*}$, which is an elliptic problem that can be solved numerically using linear algebra. Boundary conditions on $p$ in this elliptic problem depend on the specific situation considered, with the two most common being a Dirichlet condition for a constant pressure boundary condition, or a Neumann condition arising from a condition on the normal velocity component. Once $p_{n+1}$ is evaluated, Eq. 12 can then be used to calculate $\mathbf{v}_{n+1}$. A schematic representation of the method in the vector space $V_{\mathbf{v}}$ is shown in Fig. 1(a). The intermediate velocity may not be in the divergence-free subspace, but the combination of Eqs. 12 \& 13 ensures that it is projected back to this subspace.

For consistency, it is also necessary to show that the projection applied by Eq. 12 is in some sense orthogonal to the divergence-free subspace. To do this, $V_{\mathbf{v}}$ can be endowed with an inner product, where for any $\mathbf{a}, \mathbf{b} \in V_{\mathbf{v}}$,

$$
\langle\mathbf{a}, \mathbf{b}\rangle=\int \mathbf{a} \cdot \mathbf{b} d^{3} \mathbf{x}
$$

Hence, if problem-specific boundary terms are neglected, the projection $\mathbf{v}_{\mathrm{P}}=\mathbf{v}_{n+1}-\mathbf{v}_{\star}$ satisfies

$$
\left\langle\mathbf{v}_{n+1}-\mathbf{v}_{n}, \mathbf{v}_{\mathrm{P}}\right\rangle=-\frac{\Delta t}{\rho} \int\left(\mathbf{v}_{n+1}-\mathbf{v}_{n}\right) \cdot \nabla p_{n+1} d^{3} \mathbf{x}=\frac{\Delta t}{\rho} \int\left(\nabla \cdot \mathbf{v}_{n+1}-\nabla \cdot \mathbf{v}_{n}\right) p_{n+1} d^{3} \mathbf{x}=0
$$


(a)

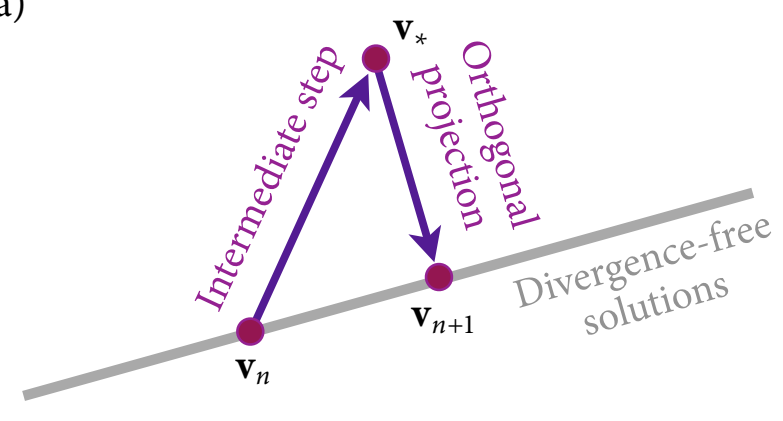

(b)

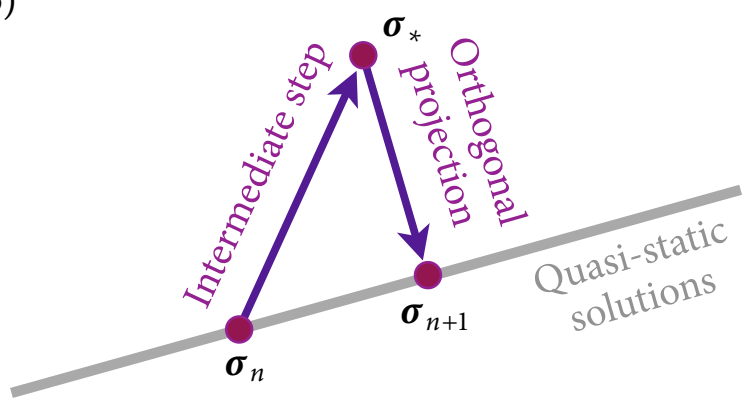

Figure 1: A schematic representation of the timestep in (a) the projection method for the incompressible Navier-Stokes equations and (b) quasi-static elastoplasticity.

and hence it is orthogonal to the divergence-free subspace. This notion of orthogonality ensures that the projection step removes the component of non-zero divergence in $\mathbf{v}_{*}$ without introducing any additional contribution to the solution in the space that is orthogonal to the projection [60], which over time could create a spurious drift in the solution.

\subsection{A projection method for quasi-static elastoplasticity}

Following the previous two sections, we conclude that there is close correspondence between the elastoplastic system and the Navier-Stokes equations for fluid flow. There is a correspondence between the variables $(\boldsymbol{\sigma}, \mathbf{v})$ in the elastoplastic system and the variables $(\mathbf{v}, p)$ for fluid flow. The limiting procedures that are employed, where the equations are scaled to examine long times, are identical.

It is therefore natural to consider whether the projection method for the incompressible NavierStokes equations can be adapted for simulating quasi-static elastoplasticity. Suppose that $\boldsymbol{\sigma}_{n}$ is a discretized stress field after $n$ timesteps, and consider making a timestep of size $\Delta t$. To begin, an intermediate stress $\sigma_{\star}$ is calculated by neglecting the total rate-of-deformation term $\mathbf{C}: \mathbf{D}$ in Eq. 1 , so that

$$
\frac{\boldsymbol{\sigma}_{*}-\boldsymbol{\sigma}_{n}}{\Delta t}=\boldsymbol{\sigma}_{n} \cdot \boldsymbol{\omega}_{n}-\boldsymbol{\omega}_{n} \cdot \boldsymbol{\sigma}_{n}-\left(\mathbf{v}_{n} \cdot \nabla\right) \boldsymbol{\sigma}_{n}-\mathbf{C}: \mathbf{D}_{n}^{\mathrm{pl}}
$$

Assuming the velocity $\mathbf{v}_{n+1}$ at the $(n+1)$ th step can be calculated, and consequently that the total deformation $\mathbf{D}_{n+1}$ is known, then the stress at the $(n+1)$ th timestep is given by

$$
\frac{\boldsymbol{\sigma}_{n+1}-\boldsymbol{\sigma}_{*}}{\Delta t}=\mathbf{C}: \mathbf{D}_{n+1} \text {. }
$$

Taking the divergence of this equation and enforcing that $\nabla \cdot \boldsymbol{\sigma}_{n+1}=\mathbf{0}$ yields

$$
\nabla \cdot \boldsymbol{\sigma}_{\star}=-\Delta t \nabla \cdot\left(\mathbf{C}: \mathbf{D}_{n+1}\right)
$$

Eq. 18 is an algebraic system for the velocity $\mathbf{v}_{n+1}$. It is analogous to Eq. 13 for the fluid projection method, and will involve second-order differential operators. It may also involve mixed derivatives, and coupling between the components of velocity, but in principle can be solved using standard numerical linear algebra techniques. As in the fluid projection method, the boundary conditions for 
$\mathbf{v}_{n+1}$ will be problem-specific, but typical cases will have simple implementations: a constant velocity boundary condition gives a Dirichlet condition on $\mathbf{v}_{n+1}$, while a traction boundary condition gives a Neumann-like condition (discussed in Sec. 5). Once $\mathbf{v}_{n+1}$ is calculated, Eq. 17 can be used to evaluate $\boldsymbol{\sigma}_{n+1}$.

A schematic representation of the algorithm is shown in Fig. 1(b) in the vector space $V_{\sigma}$ of stresses, where the quasi-static solutions form a subspace. As for the fluid projection method, it is useful to establish a notion of orthogonality by introducing an inner product. This can be constructed by making use of the compliance tensor $\mathbf{S}$, which gives the infinitesimal strain $\varepsilon$ in terms of stress according to $\boldsymbol{\varepsilon}=\mathbf{S}: \boldsymbol{\sigma}$, so that $\mathbf{S}=\mathbf{C}^{-1}$. For real materials, both $\mathbf{S}$ and $\mathbf{C}$ are positive-definite, in order to ensure that the strain energy density is positive. For two stresses $\mathbf{a}, \mathbf{b} \in V_{\boldsymbol{\sigma}}$, consider the inner product defined as

$$
\langle\mathbf{a}, \mathbf{b}\rangle=\int \mathbf{a}: \mathbf{S}: \mathbf{b} d^{3} \mathbf{x} .
$$

Since $\mathbf{S}$ is positive-definite, this will be a valid inner product. The projection $\boldsymbol{\sigma}_{\mathrm{P}}=\boldsymbol{\sigma}_{n+1}-\boldsymbol{\sigma}_{\star}$ satisfies

$$
\begin{aligned}
\left\langle\boldsymbol{\sigma}_{n+1}-\boldsymbol{\sigma}_{n}, \boldsymbol{\sigma}_{\mathrm{P}}\right\rangle & =\Delta t \int\left(\boldsymbol{\sigma}_{n+1}-\boldsymbol{\sigma}_{n}\right): \mathbf{S}:\left(\mathbf{C}: \mathbf{D}_{n+1}\right) d^{3} \mathbf{x} \\
& =\Delta t \int\left(\boldsymbol{\sigma}_{n+1}-\boldsymbol{\sigma}_{n}\right): \mathbf{D}_{n+1} d^{3} \mathbf{x}=\Delta t \int\left(\boldsymbol{\sigma}_{n+1}-\boldsymbol{\sigma}_{n}\right): \nabla \mathbf{v}_{n+1} d^{3} \mathbf{x} \\
& =-\Delta t \int\left(\nabla \cdot \boldsymbol{\sigma}_{n+1}-\nabla \cdot \boldsymbol{\sigma}_{n}\right) \cdot \mathbf{v}_{n+1} d^{3} \mathbf{x}=0,
\end{aligned}
$$

and therefore the projection is orthogonal the subspace of quasi-static solutions. For an isotropic linear elastic material with bulk modulus $K$ and shear modulus $\mu$ the components of the stiffness tensor are

$$
C_{i j k l}=\lambda \delta_{i j} \delta_{k l}+\mu\left(\delta_{i k} \delta_{j l}+\delta_{i l} \delta_{j k}\right),
$$

where $\lambda=K-\frac{2 \mu}{3}$ is Láme's first parameter. The components of the compliance tensor are

$$
S_{i j k l}=\frac{1}{6 K \mu}\left[-\lambda \delta_{i j} \delta_{k l}+\frac{3 K}{2}\left(\delta_{i k} \delta_{j l}+\delta_{i l} \delta_{j k}\right)\right] .
$$

For this case, the inner product can be written as

$$
\langle\mathbf{a}, \mathbf{b}\rangle=\frac{1}{6 K \mu} \int(3 K \mathbf{a}: \mathbf{b}-\lambda(\operatorname{tr} \mathbf{a})(\operatorname{tr} \mathbf{b})) d^{3} \mathbf{x} .
$$

As described in Appendix A, an integral argument can also be used to show that Eq. 18 has a unique solution for Dirichlet boundary conditions.

\section{A numerical implementation}

We now describe a specific finite-difference numerical implementation of the algorithms presented in Sec. 2. We make use of a rate-dependent elastoplastic model of a BMG that is based upon the STZ theory. Using this model, we test the quasi-static time-integration method against the traditional explicit scheme. All of the methods described below were implemented in a custom-written $\mathrm{C}++$ code, using the OpenMP library to multithread the loops involved in the finite-difference update. 


\subsection{Kinematics and elasticity}

A plane strain formulation in the $x$ and $y$ coordinates is used [61]. The velocity is given by $\mathbf{v}=(u, v, 0)$, and the stress tensor is written as

$$
\boldsymbol{\sigma}=\left(\begin{array}{ccc}
-p+s-q & \tau & 0 \\
\tau & -p-s-q & 0 \\
0 & 0 & -p+2 q
\end{array}\right) .
$$

Here, $p$ is the pressure, $s$ and $\tau$ are the components of deviatoric stress within the $x y$ plane, and $q$ is the component of deviatoric stress in the $z$ direction out of the plane. The deviatoric part of the stress tensor is written as $\sigma_{0}=\sigma-\frac{1}{3} 1 \operatorname{tr} \sigma$ and the magnitude of the deviatoric stress tensor is $\left|\boldsymbol{\sigma}_{0}\right|=\bar{s}=\sqrt{s^{2}+\tau^{2}+3 q^{2}}$. The density is assumed to be a constant $\rho_{0}$, since elastic deformations are assumed to be small, and the plastic deformation model is purely deviatoric. In component form, Eq. 2 reads

$$
\begin{aligned}
& \rho_{0} \frac{d u}{d t}=-\frac{\partial p}{\partial x}-\frac{\partial q}{\partial x}+\frac{\partial s}{\partial x}+\frac{\partial \tau}{\partial y}+\kappa \nabla^{2} u \\
& \rho_{0} \frac{d v}{d t}=-\frac{\partial p}{\partial y}-\frac{\partial q}{\partial y}-\frac{\partial s}{\partial y}+\frac{\partial \tau}{\partial x}+\kappa \nabla^{2} v
\end{aligned}
$$

where a small additional viscous stress term, $\kappa \nabla^{2} \mathbf{v}$ has been incorporated. This term is needed for numerical stability in the explicit simulation method. However, it is not needed for numerical stability in the quasi-static method.

The plastic deformation tensor is proportional to the deviatoric stress tensor and can therefore be written as $\mathrm{D}^{\mathrm{pl}}=\frac{\sigma_{0}}{\bar{s}} D^{\mathrm{pl}}$, where $D^{\mathrm{pl}}$ is a scalar function described in detail in the following section. In component form the constitutive equation, Eq. 1, is given by

$$
\begin{aligned}
& \frac{d p}{d t}=-K\left(\frac{\partial u}{\partial x}+\frac{\partial v}{\partial y}\right) \\
& \frac{d q}{d t}=-\frac{\mu}{3}\left(\frac{\partial u}{\partial x}+\frac{\partial v}{\partial y}\right)-\frac{2 \mu q D^{\mathrm{pl}}}{\bar{s}} \\
& \frac{d s}{d t}=2 \omega \tau+\mu\left(\frac{\partial u}{\partial x}-\frac{\partial v}{\partial y}\right)-\frac{2 \mu s D^{\mathrm{pl}}}{\bar{s}} \\
& \frac{d \tau}{d t}=-2 \omega s+\mu\left(\frac{\partial u}{\partial y}+\frac{\partial v}{\partial x}\right)-\frac{2 \mu \tau D^{\mathrm{pl}}}{\bar{s}}
\end{aligned}
$$

where $\omega=(\partial v / \partial x-\partial u / \partial y) / 2, K$ is the bulk modulus, and $\mu$ is the shear modulus. Table 1 shows the values of the elastic parameters used in this study, which are based on Vitreloy 1, a specific type of BMG whose mechanical properties have been well-studied.

\subsection{Plasticity}

Plastic deformation is modeled using the shear transformation zone theory of amorphous plasticity $[47,62]$. We employ a version of the model used to study fracture [57], which is based on recent 


\begin{tabular}{l|l} 
Parameter & Value \\
\hline Young's modulus $E$ & $101 \mathrm{GPa}$ \\
Poisson ratio $v$ & 0.35 \\
Bulk modulus $K$ & $122 \mathrm{GPa}$ \\
Shear modulus $\mu$ & $37.4 \mathrm{GPa}$ \\
Density $\rho_{0}$ & $6125 \mathrm{~kg} \mathrm{~m}^{-3}$ \\
Shear wave speed $c_{s}=\sqrt{\mu / \rho_{0}}$ & $2.47 \mathrm{~km} \mathrm{~s}^{-1}$
\end{tabular}

Table 1: Elasticity parameters used throughout the paper.

\begin{tabular}{l|l} 
Parameter & Value \\
\hline Yield stress $s_{\mathrm{Y}}$ & $0.85 \mathrm{GPa}$ \\
Molecular vibration timescale $\tau_{0}$ & $10^{-13} \mathrm{~s}$ \\
Typical local strain $\varepsilon_{0}$ & 0.3 \\
Scaling parameter $c_{0}$ & 0.4 \\
Typical activation barrier $\Delta / k_{B}$ & $8000 \mathrm{~K}$ \\
Typical activation volume $\Omega$ & $300 \AA^{3}$ \\
Bath temperature $T$ & $400 \mathrm{~K}$ \\
Steady state effective temperature $\chi_{\infty}$ & $900 \mathrm{~K}$ \\
STZ formation energy $e_{z} / k_{B}$ & $21000 \mathrm{~K}$
\end{tabular}

Table 2: Parameter values for the STZ plasticity model used throughout the paper. The Boltzmann constant $k_{B}=$ $1.3806488 \times 10^{-23} \mathrm{~J} \mathrm{~K}^{-1}$ is used to express the quantities $\Delta$ and $e_{Z}$ in terms of temperature.

theoretical developments $[49,63]$, although simplified to retain only the crucial details. Here, we sketch the theoretical principles behind the model and provide the relevant equations.

Consider a $B M G$ at a temperature $T$ below the glass transition temperature. If no stress is applied, then the constituent atoms will undergo thermal vibrations, but will largely remain in the same overall packing configuration with their neighbors; in terms of an energy landscape, they are trapped within a potential well representing one mechanically stable configuration. If the BMG is subjected to a shear stress, then discrete events will occur whereby some atoms in a local region undergo an irreversible change in configuration-the applied stress changes the energy landscape to lower the potential barrier of the well, so that it becomes possible to jump to another well representing a different mechanically stable configuration.

This physical picture can be used to derive a continuum plasticity model. One imagines that the material has a population of shear transformation zones, which represent localized regions that are susceptible to shear-driven configurational changes. The density of STZs is described in terms of an effective disorder temperature $\chi$. For $\bar{s}<s_{\mathrm{Y}}$, where $s_{\mathrm{Y}}$ is the yield stress of the material, the plastic deformation is zero. For $\bar{s} \geq s_{\mathrm{Y}}$, the plastic deformation is given by

$$
D^{\mathrm{pl}}\left(\boldsymbol{\sigma}_{0}, T, \chi\right)=\frac{\Lambda(\chi) \mathcal{C}(\bar{s}, T)}{\tau_{0}}\left(1-\frac{s_{\mathrm{Y}}}{\bar{s}}\right),
$$

where $\tau_{0}$ is a molecular vibration timescale, $\mathcal{C}(\bar{s}, T)$ is the STZ transition rate, and $\Lambda(\chi)=e^{-e_{z} / k_{B} \chi}$ is the density of STZs in terms of effective temperature, where $e_{z}$ is the STZ formation energy and $k_{B}$ is 
the Boltzmann constant. The function $\mathcal{C}(\bar{s}, T)$ is specified in terms of the forward and backward STZ transition rates,

$$
\mathcal{C}(\bar{s}, T)=\frac{1}{2}(\mathcal{R}(\bar{s}, T)+\mathcal{R}(-\bar{s}, T)),
$$

which follow a linearly stress-biased thermal activation process

$$
\mathcal{R}( \pm \bar{s}, T)=\exp \left(-\frac{\Delta \mp \Omega \varepsilon_{0} \bar{s}}{k_{B} T}\right)
$$

where $\Delta$ is a typical energy activation barrier, $\Omega$ is a typical activation volume, and $\varepsilon_{0}$ is a typical local strain at the transition. Substituting Eq. 33 into Eq. 32 yields

$$
\mathcal{C}(\bar{s}, T)=e^{-\Delta / k_{B} T} \cosh \frac{\Omega \varepsilon_{0} \bar{s}}{k_{B} T} .
$$

For very large positive values of $\bar{s}$, it is possible that the stress-biasing $\Omega \varepsilon_{0} \bar{s}$ will exceed the activation barrier $\Delta$, in which case the physical picture of a thermally activated process is no longer valid. In previous work, we have assumed that for $\bar{s} \Omega \varepsilon_{0} \geq \Delta$ the plastic behavior is dominated by a different, weaker, dissipative mechanism $[62,36]$. However, we omit this term here for mathematical simplicity. For the parameters given in Table 2 the barrier is reached at $\bar{s}=1.44 s_{\mathrm{Y}}$, and apart from the final example in Subsec. 5.5 where this issue is considered in more detail, the deviatoric stresses never exceed $1.35 s_{\mathrm{Y}}$, since the exponential growth of $D^{\mathrm{pl}}$ as a function of $\bar{s}$ causes large deviatoric stresses to rapidly relax. The effective temperature follows a heat equation of the form

$$
c_{0} \frac{d \chi}{d t}=\frac{\left(\mathrm{D}^{\mathrm{pl}}: \boldsymbol{\sigma}_{0}\right)\left(\chi_{\infty}-\chi\right)}{s_{\mathrm{Y}}}
$$

so that $\chi$ increases in response to plastic deformation and saturates at $\chi_{\infty}$. Since an increase in $\chi$ will also increase $D^{\mathrm{pl}}$ as given by Eq. 31, the plasticity model typically leads to shear banding $[64,65]$.

\subsection{Numerical methods for explicit simulations}

The simulations are carried out on a rectangular $M \times N$ grid of square cells with side length $h$. As shown in Fig. 2(a), a staggered arrangement is used whereby the components of velocity $u, v$ are stored at cell corners and indexed with integers, and the components of stress $p, q, s, \tau$ and effective temperature $\chi$ are stored at cell centers and indexed with half-integers. The explicit simulation method employs Eqs. 25 to 30 and Eq. 35 to explicitly update all the simulation fields, using a first-order temporal discretization and a second-order spatial discretization.

The first derivatives on the right hand sides of Eqs. 25 to 30 are evaluated using centered differencing. It can be observed that the equations for velocity depend on first derivatives of stress and vice versa. If $f_{i, j}$ represents one of the discretized fields at a given instant, then the staggered first derivative in the $x$ direction is evaluated as

$$
\left[\frac{\partial f}{\partial x}\right]_{i+\frac{1}{2}, j+\frac{1}{2}}=\frac{f_{i+1, j}+f_{i+1, j+1}-f_{i, j}-f_{i, j+1}}{2 h} .
$$



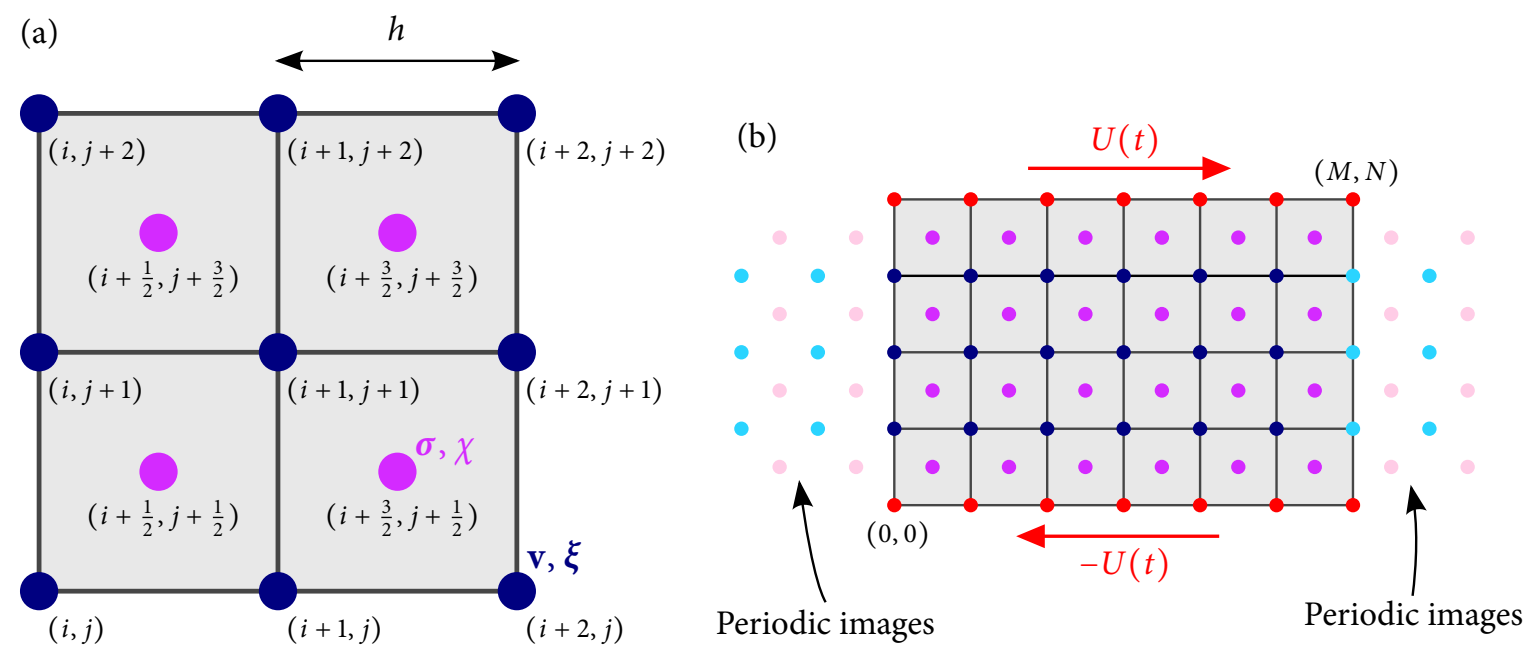

Figure 2: (a) Arrangement of fields in the spatial discretization. The simulation is divided into square cells of side length $h$. The velocity $\mathbf{v}$ and reference map $\xi$ are stored at cell corners (dark blue), which are indexed with integers. The stress tensor $\boldsymbol{\sigma}$ and effective temperature $\chi$ are stored at cell centers (magenta), which are indexed with half-integers. (b) Grid arrangement in the shearing simulation. The velocity in the top and bottom rows (red) of the simulation is fixed to create simple shear. To enforce the periodic boundary conditions in the horizontal $x$ direction, periodic images for both the cell-centered (pink) and cell-cornered (light blue) fields are used. In the example shown, $(M, N)=(6,4)$.

The viscosity terms make use of a colocated second-order derivative, which is evaluated in the $x$ direction as

$$
\left[\frac{\partial^{2} f}{\partial x^{2}}\right]_{i, j}=\frac{f_{i+1, j}-2 f_{i, j}+f_{i-1, j}}{h^{2}}
$$

The advective derivatives on the left hand side of Eqs. 25 to 30 need to be upwinded for stability. This is achieved by using the second-order ENO numerical scheme [66], which in the $x$ direction is given by

$$
\left\{\frac{\partial f}{\partial x}\right\}_{i, j}=\frac{1}{2 h} \begin{cases}-f_{i+2, j}+4 f_{i+1, j}-3 f_{i, j} & \text { if } u_{i, j}<0 \text { and }\left|\left[f_{x x}\right]_{i, j}\right|>\left|\left[f_{x x}\right]_{i+1, j}\right|, \\ 3 f_{i, j}-4 f_{i-1, j}+f_{i-2, j} & \text { if } u_{i, j}>0 \text { and }\left|\left[f_{x x}\right]_{i, j}\right|>\left|\left[f_{x x}\right]_{i-1, j}\right|, \\ f_{i+1, j}-f_{i-1, j} & \text { otherwise, }\end{cases}
$$

where $\left[f_{x x}\right]_{i, j}$ is the second-order centered-difference at $i, j$ evaluated using Eq. 37. The ENO derivative therefore switches between an upwinded one-sided derivative and a centered derivative, depending on which set of three field values is more colinear. In the $y$ direction, analogous expressions to Eq. 36 and Eq. 38 are used.

The first-order forward Euler scheme is used for timestepping. If velocity components and pressure at timestep $n$ are written as $u_{n}, v_{n}$, and $p_{n}$, and a timestep $\Delta t$ is taken, then at timestep 
$(n+1)$ they are given by

$$
\begin{aligned}
\rho_{0} \frac{u_{n+1}-u_{n}}{\Delta t} & =-\rho_{0}\left(\mathbf{v}_{n} \cdot \nabla\right) u_{n}-\frac{\partial p_{n}}{\partial x}-\frac{\partial q_{n}}{\partial x}+\frac{\partial s_{n}}{\partial x}+\frac{\partial \tau_{n}}{\partial y}+\kappa \nabla^{2} u_{n} \\
\rho_{0} \frac{v_{n+1}-v_{n}}{\Delta t} & =-\rho_{0}\left(\mathbf{v}_{n} \cdot \nabla\right) v_{n}-\frac{\partial p_{n}}{\partial y}-\frac{\partial q_{n}}{\partial y}-\frac{\partial s_{n}}{\partial y}+\frac{\partial \tau_{n}}{\partial x}+\kappa \nabla^{2} v_{n} \\
\frac{p_{n+1}-p_{n}}{\Delta t} & =-\left(\mathbf{v}_{n} \cdot \nabla\right) p_{n}-K\left(\frac{\partial u_{n}}{\partial x}+\frac{\partial v_{n}}{\partial y}\right) .
\end{aligned}
$$

The deviatoric stress components are updated with a similar procedure, but make use of a modification to accommodate for the rapid growth of $D^{\mathrm{pl}}$ when $\bar{s}$ exceeds the yield stress $s_{\mathrm{Y}}$, which causes a loss of accuracy if $\Delta t$ is too large. Suppose that at a given location and timestep, a discretized deviatoric stress $\bar{s}_{n}$ is slightly above $s_{Y}$. Physically, plastic deformation should cause the deviatoric stress to decrease until reaching the yield surface so that $\bar{s}_{n+1} \approx s_{\mathrm{Y}}$. However, if other terms are neglected, then the Euler step will give $\bar{s}_{n+1}=\bar{s}_{n}-2 \mu D^{\mathrm{pl}} \Delta t / s_{\mathrm{Y}}$ at the next timestep, which could be significantly lower than $s_{\mathrm{Y}}$ if $D^{\mathrm{pl}}$ is large, overshooting the yield surface. To solve this, an adaptive timestepping routine is used that divides the interval $\Delta t$ into subintervals so that the incremental changes to $\bar{s}$ remain small-this accomplishes a similar goal as the return-mapping algorithms for rate-independent plasticity $[41,46]$. The routine, described in Appendix B, considers the coupled system $\bar{s}$ and $\chi$ and returns modified functions $\tilde{D}_{n}^{\mathrm{pl}}$ and $\tilde{F}_{n}$ for use in the finite-difference update. The deviatoric stress and effective temperature are updated according to

$$
\begin{aligned}
& \frac{q_{n+1}-q_{n}}{\Delta t}=-\left(\mathbf{v}_{n} \cdot \nabla\right) q_{n}-\frac{\mu}{3}\left(\frac{\partial u_{n}}{\partial x}+\frac{\partial v_{n}}{\partial y}\right)-\frac{2 \mu \tilde{D}_{n}^{\mathrm{pl}} q_{n}}{\bar{s}_{n}}, \\
& \frac{s_{n+1}-s_{n}}{\Delta t}=-\left(\mathbf{v}_{n} \cdot \nabla\right) s_{n}+2 \omega_{n} \tau_{n}+\mu\left(\frac{\partial u_{n}}{\partial x}-\frac{\partial v_{n}}{\partial y}\right)-\frac{2 \mu \tilde{D}_{n}^{\mathrm{pl}} s_{n}}{\bar{s}_{n}}, \\
& \frac{\tau_{n+1}-\tau_{n}}{\Delta t}=-\left(\mathbf{v}_{n} \cdot \nabla\right) \tau_{n}-2 \omega_{n} s_{n}+\mu\left(\frac{\partial u_{n}}{\partial y}+\frac{\partial v_{n}}{\partial x}\right)-\frac{2 \mu \tilde{D}_{n}^{\mathrm{pl}} \tau_{n}}{\bar{s}_{n}}, \\
& \frac{\chi_{n+1}-\chi_{n}}{\Delta t}=-\left(\mathbf{v}_{n} \cdot \nabla\right) \chi_{n}-\tilde{F}_{n},
\end{aligned}
$$

where $\omega_{n}=\left(\partial v_{n} / \partial x-\partial u_{n} / \partial y\right) / 2$ and the $\mathbf{v}_{n}$ term in the advective derivatives is evaluated as the average of the velocities at the four corners of the grid cell.

The simulation also makes use of a reference map vector field $\xi=\left(\xi^{x}, \xi^{y}\right)$ stored at cell corners. This field has no physical influence, but is used to track the deformation of the material. It is initialized as

$$
\boldsymbol{\xi}(\mathbf{x}, 0)=\mathbf{x}
$$

and is then updated according to

$$
\frac{d \boldsymbol{\xi}}{d t}=\frac{\partial \boldsymbol{\xi}}{\partial t}+(\mathbf{v} \cdot \nabla) \boldsymbol{\xi}=\mathbf{0}
$$


following the same discretization methods as for the other fields. Contours of the components of the reference map initially form a rectangular grid and then deform with the material. Using $\xi$, the $(2 \times 2)$-component deformation gradient tensor is given by

$$
\mathbf{F}=\frac{\partial \mathbf{x}}{\partial \xi}
$$

which can be numerically evaluated using centered differences of $\boldsymbol{\xi}$. Once $\mathbf{F}$ is known, the GreenSaint-Venant strain tensor is given by $\mathbf{E}=\frac{1}{2}\left(\mathbf{F}^{\top} \mathbf{F}-\mathbf{1}\right)$. The deviatoric part of the strain tensor is defined as $\mathbf{E}_{0}=\mathbf{E}-\frac{1}{2} \mathbf{1} \operatorname{tr} \mathbf{E}$.

\subsection{Numerical methods for quasi-static simulations}

The quasi-static scheme makes use of the same simulation framework as the explicit scheme. It employs the same rectangular grid, and uses Eqs. 36 and 38 for carrying out spatial derivatives. To carry out a timestep of size $\Delta t$, Eq. 16 is first used to calculate in intermediate stress $\sigma_{\star}$, which in component form is

$$
\begin{aligned}
& \frac{p_{*}-p_{n}}{\Delta t}=-\left(\mathbf{v}_{n} \cdot \nabla\right) p_{n}, \\
& \frac{q_{*}-q_{n}}{\Delta t}=-\left(\mathbf{v}_{n} \cdot \nabla\right) q_{n}-\frac{2 \mu \tilde{D}_{n}^{\mathrm{pl}} q_{n}}{\bar{s}_{n}}, \\
& \frac{s_{*}-s_{n}}{\Delta t}=-\left(\mathbf{v}_{n} \cdot \nabla\right) s_{n}+2 \Omega_{n} \tau_{n}-\frac{2 \mu \tilde{D}_{n}^{\mathrm{pl}} s_{n}}{\bar{s}_{n}}, \\
& \frac{\tau_{*}-\tau_{n}}{\Delta t}=-\left(\mathbf{v}_{n} \cdot \nabla\right) \tau_{n}-2 \Omega_{n} s_{n}-\frac{2 \mu \tilde{D}_{n}^{\mathrm{pl}} \tau_{n}}{\bar{s}_{n}} .
\end{aligned}
$$

The adaptive prodecure described in Appendix B is used to evaluate the plastic deformation term $\tilde{D}_{n}^{\mathrm{pl}}$ that features in these equations. It also returns $\tilde{F}_{n}$, which allows $\chi_{n+1}$ to be calculated according to Eq. 45.

If the velocity $\mathbf{v}_{n+1}$ at timestep $n+1$ is known, then by following Eq. 17, the components of $\boldsymbol{\sigma}_{n+1}$ are given by

$$
\begin{aligned}
\frac{p_{n+1}-p_{*}}{\Delta t} & =-K\left(\frac{\partial u_{n+1}}{\partial x}+\frac{\partial v_{n+1}}{\partial y}\right), \\
\frac{q_{n+1}-q_{*}}{\Delta t} & =-\frac{\mu}{3}\left(\frac{\partial u_{n+1}}{\partial x}+\frac{\partial v_{n+1}}{\partial y}\right), \\
\frac{s_{n+1}-s_{*}}{\Delta t} & =\mu\left(\frac{\partial u_{n+1}}{\partial x}-\frac{\partial v_{n+1}}{\partial y}\right), \\
\frac{\tau_{n+1}-\tau_{*}}{\Delta t} & =\mu\left(\frac{\partial u_{n+1}}{\partial y}+\frac{\partial v_{n+1}}{\partial x}\right) .
\end{aligned}
$$


To calculate $\mathbf{v}_{n+1}$, the quasi-staticity constraint at the $(n+1)$ th timestep is used, which by retaining the viscous stress is slightly modified to $\mathbf{0}=\nabla \cdot \boldsymbol{\sigma}_{n+1}+\kappa \nabla^{2} \mathbf{v}_{n+1}$. Following Eq. 18, the velocity satisfies

$$
\begin{aligned}
& \left(\mu+K^{\prime}+\kappa^{\prime}\right) \frac{\partial^{2} u_{n+1}}{\partial x^{2}}+\left(\mu+\kappa^{\prime}\right) \frac{\partial^{2} u_{n+1}}{\partial y^{2}}+K^{\prime} \frac{\partial^{2} v_{n+1}}{\partial x \partial y}=\frac{1}{\Delta t}\left(\frac{\partial p_{*}}{\partial x}+\frac{\partial q_{\star}}{\partial x}-\frac{\partial s_{*}}{\partial x}-\frac{\partial \tau_{\star}}{\partial y}\right) \\
& \left(\mu+\kappa^{\prime}\right) \frac{\partial^{2} v_{n+1}}{\partial x^{2}}+\left(\mu+K^{\prime}+\kappa^{\prime}\right) \frac{\partial^{2} v_{n+1}}{\partial y^{2}}+K^{\prime} \frac{\partial^{2} u_{n+1}}{\partial x \partial y}=\frac{1}{\Delta t}\left(\frac{\partial p_{\star}}{\partial y}+\frac{\partial q_{\star}}{\partial y}+\frac{\partial s_{\star}}{\partial y}-\frac{\partial \tau_{\star}}{\partial x}\right)
\end{aligned}
$$

where $K^{\prime}=K+\frac{\mu}{3}$ and $\kappa^{\prime}=\frac{\kappa}{\Delta t}$. In the typical regime of interest where $\Delta t$ becomes large, the effect of the viscous term is therefore negligible.

Eqs. 57 and 58 form an algebraic system for the components of velocity. The system features second derivatives and bears some similarity to the Poisson equation that must be solved for the fluid projection method. However, the system is more complicated, since the two components of velocity are coupled, and a mixed $x y$-derivative is present. To solve the equations, a linear system $A_{0}$ is constructed where the derivatives are discretized using Eqs. 36 \& 37, and

$$
\left[\frac{\partial^{2} f}{\partial x \partial y}\right]_{i, j}=\frac{f_{i+1, j+1}-f_{i+1, j-1}-f_{i-1, j+1}+f_{i-1, j-1}}{4 h^{2}}
$$

where $f_{i, j}$ represents the components of an arbitrary field. The linear system also takes into account problem-specific boundary conditions, which are discussed later.

The presence of the mixed derivative means that the linear system is not weakly diagonally dominant, unlike the Poisson problem for the fluid projection method. However, in general as discussed previously, the matrix will be symmetric and positive-definite, other than possible complications due to the application of boundary conditions. The linear system is therefore well-suited to be solved by many linear algebra techniques and will admit a unique solution. For the cases considered here, the linear system is solved using a custom-written geometric multigrid algorithm.

\section{Shearing between two parallel plates}

The first example considered is a material being sheared between two parallel plates. This example has simple boundary conditions, but exhibits complex behavior and shear banding, making it a useful environment in which to compare the explicit and quasi-static simulation approaches. The example uses a domain that is periodic in the $x$ direction and covers $-\gamma L<x \leq \gamma L,-L \leq y \leq L$ where $\gamma$ is a dimensionless constant. Initially, the velocity and Cauchy stress are zero, and the reference map is given Eq. 46. A natural time unit is $t_{s}=L / c_{s}$. The boundary conditions on the top and bottom boundaries are

$$
\mathbf{v}(x, \pm L, t)=( \pm U(t), 0),\left.\quad \frac{\partial \boldsymbol{\sigma}}{\partial y}\right|_{y= \pm L}=\left.\frac{\partial \chi}{\partial y}\right|_{y= \pm L}=0, \quad \xi(x, \pm L, t)=(x \mp X(t), \pm L),
$$

where the function $U(t)$ satisfies

$$
U(t)= \begin{cases}\frac{U_{\mathrm{B}} t}{t_{s}} & \text { for } t<t_{s} \\ U_{\mathrm{B}} & \text { for } t \geq t_{s}\end{cases}
$$


so that the speed of the parallel plates is linearly increased to a value $U_{\mathrm{B}}$, after which it remains constant. This form for $U(t)$ causes the stresses in the material to gradually increase, and avoids the problem that applying $U(t)=U_{\mathrm{B}}$ for $t>0$ would immediately create a very large deformation rate next to the boundaries. For consistency, the function $X(t)$ in Eq. 59 is given by

$$
X(t)=\int_{0}^{t} U\left(t^{\prime}\right) d t^{\prime}= \begin{cases}\frac{U_{\mathrm{B}} t^{2}}{2 t_{s}} & \text { for } t<t_{s} \\ U_{\mathrm{B}}\left(\frac{t_{s}}{2}+t\right) & \text { for } t \geq t_{s}\end{cases}
$$

A schematic of the grid point layout is shown in Fig. 2(b). The cell-cornered grid points $(i, j)$ cover the index ranges $i=0,1, \ldots, M-1$ and $j=0,1, \ldots, N$, and cell-centered grid points cover the index ranges $i=\frac{1}{2}, \frac{3}{2}, \ldots, \frac{2 M-1}{2}$ and $j=\frac{1}{2}, \frac{3}{2}, \ldots, \frac{2 N-1}{2}$. The location of grid point $(i, j)$ is at $(x, y)=(-\gamma L+h i,-L+h j)$ so that $j=0$ is located on the bottom boundary and $j=N$ is located on the top boundary. Throughout the simulation, the field values for $j=0$ and $j=N$ are set using the boundary conditions in Eq. 59.

Explicit and quasi-static simulations are carried out using the methods described in Subsecs. 3.3 and 3.4 respectively, and are applied to grid points in the range $\frac{1}{2} \leq j \leq \frac{2 N-1}{2}$. To handle the periodic boundary conditions, the spatial finite-difference operators wrap around; for example, a reference to an arbitrary field value $f_{M, j}$ is treated as $f_{0, j}$. In addition, a displacement of $2 \gamma L$ is applied to the $x$-component of the reference map, so that $\xi_{M, j}^{x}=\xi_{0}^{x}+2 \gamma L$. When calculating upwinded derivatives in the $y$-direction at $j=\frac{1}{2}, 1$ and $j=n-1, \frac{2 n-1}{2}$ using Eq. 38, the simulation falls back on a first-order upwinded derivative if not enough grid points are available to calculate the ENO discretization. For this example, the algebraic problem considered in the quasi-static simulation method is simple to implement and makes use of Dirichlet conditions on $\mathbf{v}$ at $j=0$ and $j=N$.

\subsection{Comparison of explicit and quasi-static methods}

We first consider a case where the parameters are chosen to allow for a quantitative comparison between the explicit and quasi-static simulation approaches. We make use of $L=1 \mathrm{~cm}, \gamma=4$, and consider an initial effective temperature distribution of the form

$$
\chi(\mathbf{x}, t)=630 \mathrm{~K}+(170 \mathrm{~K}) \exp \left(-\frac{|20 \mathbf{x}|^{2}}{2 L^{2}}\right)
$$

corresponding to a small imperfection in the center of the domain. When subjected to shear, we expect that a shear band will nucleate from the imperfection, creating a region where a plastic deformation will be localized. The parameters given in Tables 1 and 2 are used as a baseline, and for the given value of $L$, the natural timescale is $t_{s}=4.05 \mu \mathrm{s}$. A grid size of $640 \times 160$ is used, so that the grid spacing is $h=\frac{L}{80}$.

To quantitatively compare the explicit and quasi-static simulation approaches, a parameter $\zeta$ is introduced that can control the overall speed of the dynamics in a manner similar to the scaling argument in Eq. 3. The boundary speed is set to $U_{\mathrm{B}}=10^{-7} \zeta \mathrm{L} / t_{\mathrm{s}}=247 \zeta \mu \mathrm{m} / \mathrm{s}$ and the plastic deformation rate is scaled by $\zeta$, by replacing $\tau_{0}$ with $10^{-13} \zeta^{-1} \mathrm{~s}^{-1}$. Simulations over a duration of $2 \times 10^{6} t_{s} \zeta^{-1}=8.06 \zeta^{-1} \mathrm{~s}$ are carried out, after which the boundaries are each displaced by approximately $2 \mathrm{~mm}$. For $\zeta=1$, the scales are approximately in physically reasonable ranges for typical experimental 


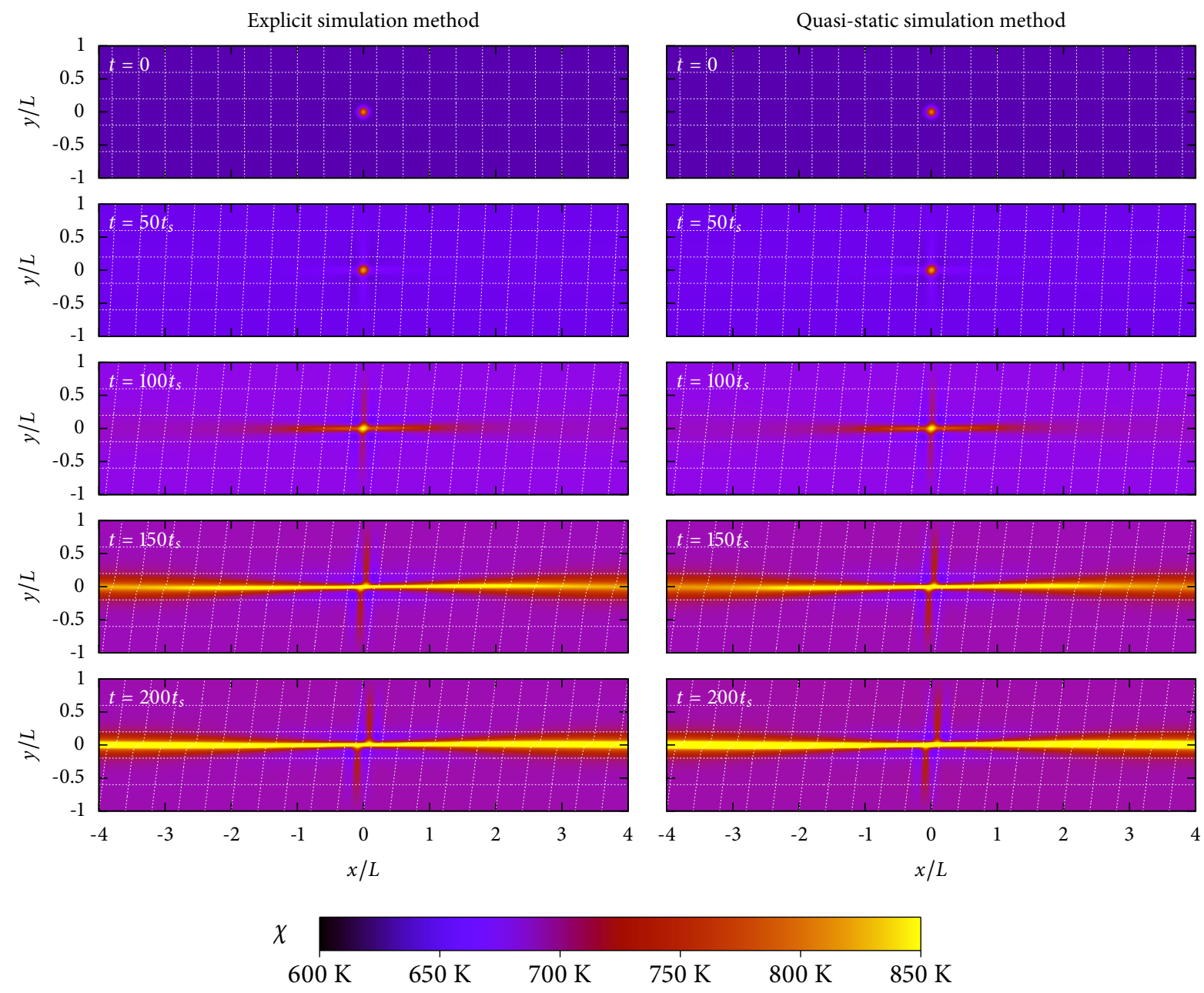

Figure 3: Plots of effective temperature $\chi$ at five time points for the shear band nucleation simulation, using the explicit simulation method (left) and the quasi-static simulation method (right). The thin dashed white lines are the contours of the components of the reference map $\xi$, and show how the material deforms. As described in the text, the simulation is speeded up by a factor of $\zeta=10^{4}$ from physical parameters to make it computationally feasible to compare the two numerical methods. 


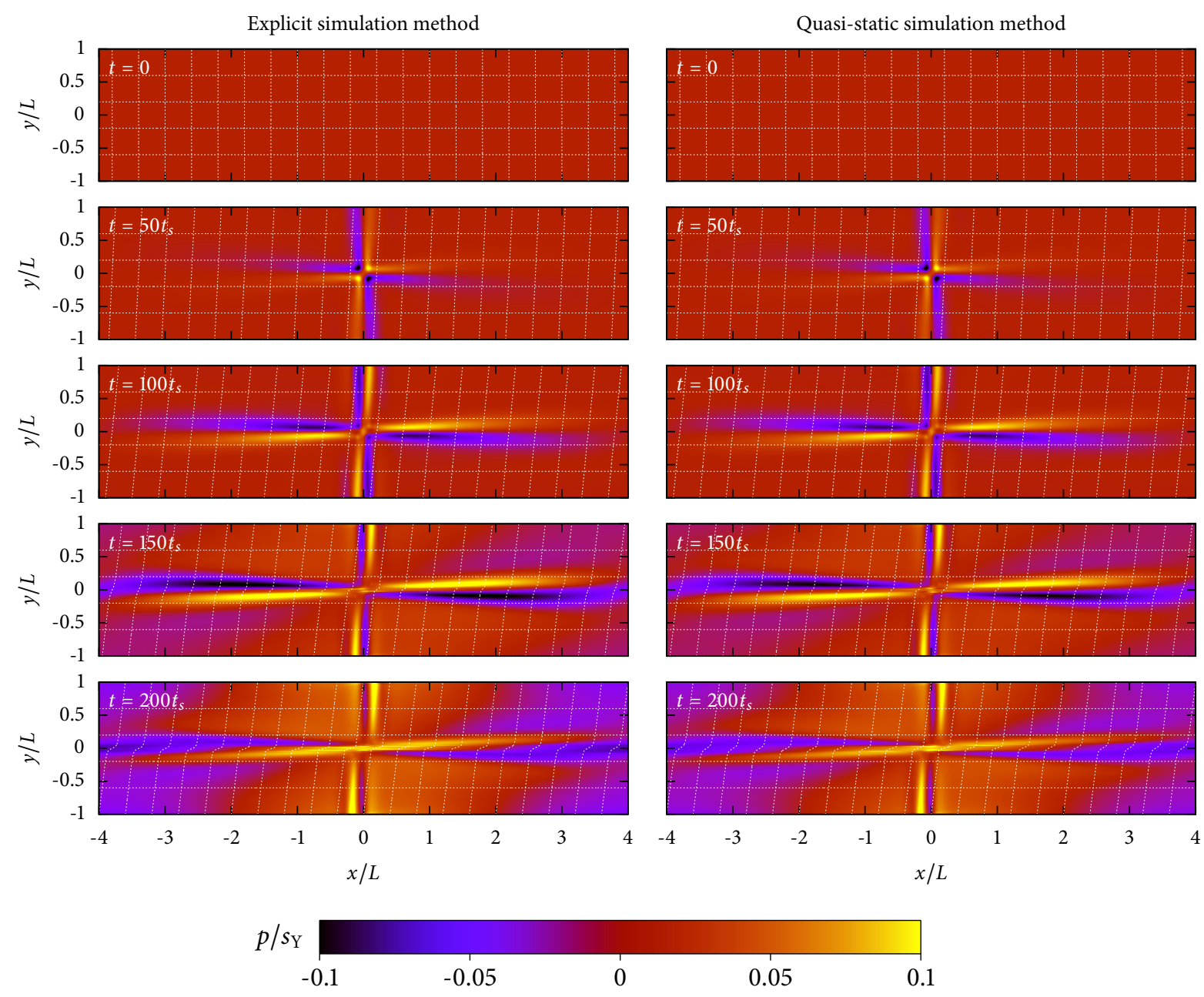

Figure 4: Plots of pressure $p$ at five time points for the shear band nucleation simulation, using the explicit simulation method (left) and the quasi-static simulation method (right). The thin dashed white lines are the contours of the components of the reference map $\xi$, and show how the material is deforms. The simulation is speeded up by a factor of $\zeta=10^{4}$ from physical parameters.

tests. The timestep used in the explicit simulation is $\Delta t=\frac{t_{s} h^{2}}{2 L^{2}}$ so that the viscous stress can be properly resolved. The timestep used in the quasi-static simulation is $\Delta t=\frac{t_{s} \zeta}{50}$ respectively.

Figure 3 shows a sequence of snapshots of effective temperature, for both the explicit simulation and the quasi-static simulation, using an artificial scaling factor of $\zeta=10^{4}$. The two simulation methods give very similar results and are hard to differentiate by eye. At $t=50 t_{s}$, the effective temperature has increased uniformly by a small amount throughout the material, but bands of slightly higher $\chi$ have begun to emerge in the orthogonal directions from the initial imperfection. By $t=100 t_{s}$, the horizontal band starts to dominate, and by $t=150 t_{s}$ it has grown across the entire width of the simulation. The shear band continues to grow larger by $t=200 t_{s}$, and accommodates most of the plastic deformation.

When the full shear band initially forms at $t \approx 150 t_{s}$, it is approximately three simulation grid 


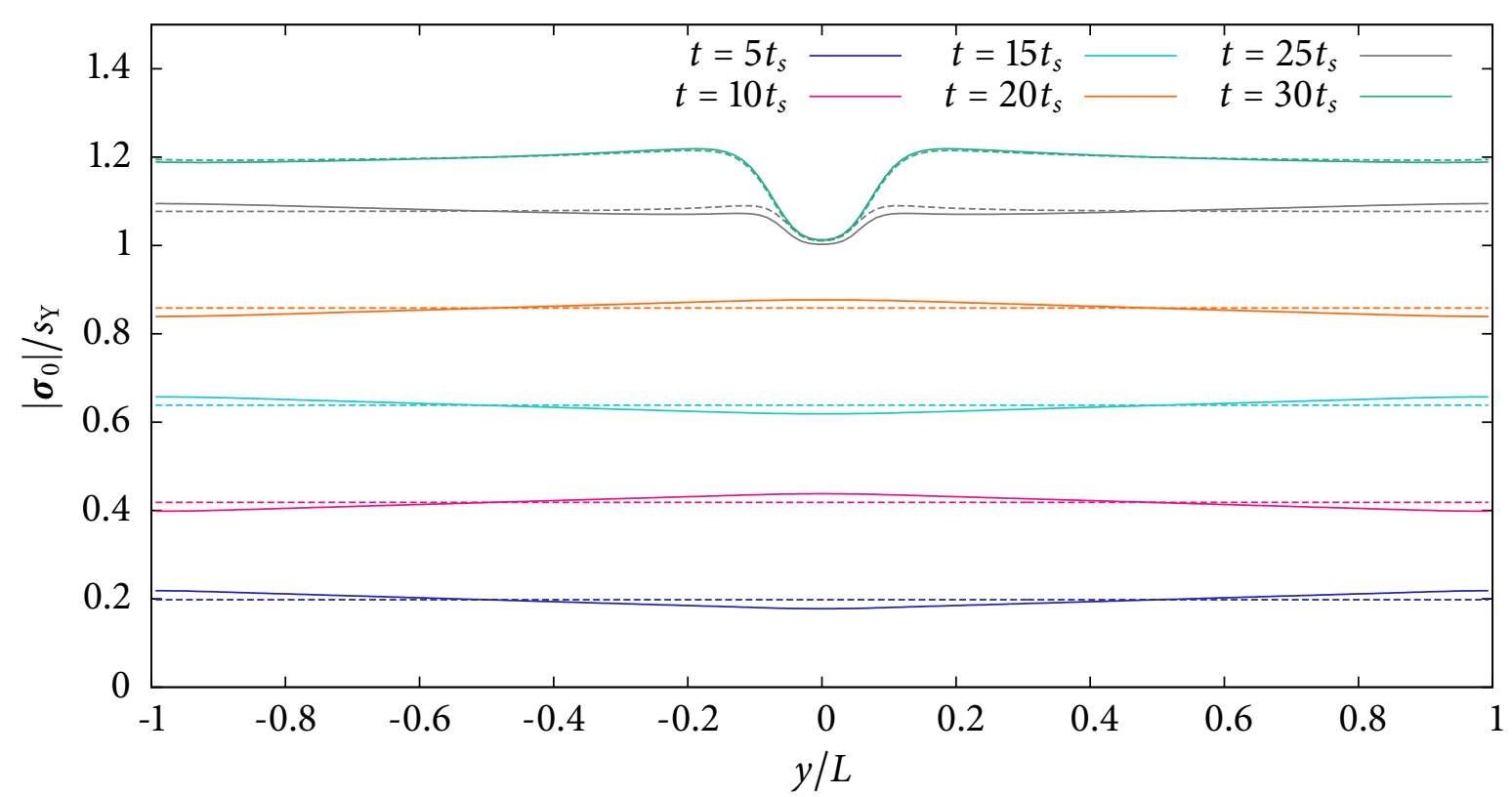

Figure 5: Comparison of the cross-sections of the deviatoric stress field on the line $y=0$ for the explicit shear band simulation (solid lines) and the quasi-static shear band simulation (dashed lines).

points across, and may therefore not be fully resolved; its width may partly be governed by numerical diffusion. At later times as more plastic deformation occurs, the shear band width continues to grow, consistent with one-dimensional studies [64]. Figure 4 shows plots of the pressure field for the two simulation method, at the same sequence of time points. The pressure deviations are relatively small, reaching values up to $\frac{1}{10} s_{\mathrm{Y}}$, but again there is very good agreement between the two methods. The increased plastic deformation near the initial imperfection leads to a small quadrupolar feature the pressure field.

Figure 5 shows the cross sections of the deviatoric stress $\left|\boldsymbol{\sigma}_{0}\right|$ for the two simulations, for several time points up to $t=30 t_{s}$. The graph highlights some small differences between the methods. In the quasi-static simulation, $\left|\sigma_{0}\right|$ is uniform in $y$ up to $t=20 t_{s}$ while the material is in the elastic regime and the shear stress are below the yield stress. The corresponding plots for the explicit simulation are similar, although show slight oscillations, due to elastic waves propagating across the material. Even though the shearing velocity is gradually increased following Eq. 60, some small elastic waves are introduced at the start of the simulation, which continue to propagate across the simulation since there is little damping to remove them. By $t=25 t_{s}$ some plastic deformation starts to occur resulting in a reduction of shear stress near $y=0$. Since the plastic deformation introduces some dissipation, the elastic waves in the explicit simulation are damped out, meaning that by $t=30 t_{s}$ the two simulation methods come into closer agreement.

These simulations were carried out using eight threads on a Mac Pro (Late 2013) with an 8-core $3 \mathrm{GHz}$ Intel Xeon E5 processor. The explicit simulation used 2,560,000 timesteps, took a total wall clock time of $8020 \mathrm{~s}$, corresponding to an average wall clock time of $3.13 \mathrm{~ms}$ per integration step. The quasi-static simulation used 20,000 timesteps, took a total wall clock time of $1370 \mathrm{~s}$, corresponding 
to an average wall clock time of $68.6 \mathrm{~ms}$ per integration step. While the quasi-static simulation step takes more than twenty times longer than the explicit timestep due to solving a linear system using the multigrid method, its ability to take much larger steps means that the total simulation time is about a sixth that of the explicit simulation. At lower values of $\zeta$, the quasi-static simulation will require the same computation time, while the computation time for the explicit simulation take longer, since the time required is inversely proportional to $\zeta$.

\subsection{Quantitative comparison of the explicit and quasi-static simulation methods}

The quasi-static system of equations given by Eqs. 1 and 6 emerges from taking a limit of slow velocity and long times, and in this section we quantitatively compare the two simulation methods in this limit. We employ the same boundary conditions as in the previous section, and we expect that as $\zeta$ is reduced, the differences between the two methods will tend to zero. However, quantitatively examining this poses some difficulties, since in addition to simulating different equations, the two methods introduce different discretization errors. It is therefore necessary to consider additional parameters that affect the discretization.

To evaluate the differences between the explicit and quasi-static simulations, a norm

$$
\|\mathbf{f}\|=\sqrt{\frac{1}{16 L^{2}} \int_{-4 L}^{4 L} d x \int_{-L}^{L}|\mathbf{f}|^{2} d y}
$$

is introduced where $\mathbf{f}$ is an arbitrary field, and the integrals are evaluated using the trapezoidal rule. By interpreting $|\mathbf{f}|^{2}$ appropriately, Eq. 63 can be applied to scalars, vectors, and tensors. To create more of a spread in the effective temperature field, we consider an alternative initial condition corresponding to rotated line of higher $\chi$. The function

$$
\Gamma\left(x^{\prime}, y^{\prime}\right)= \begin{cases}\exp \left(-\frac{\left|20 y^{\prime}\right|^{2}}{2 L^{2}}\right) & \text { if }\left|x^{\prime}\right| \leq L, \\ \exp \left(-\frac{\mid 400\left(\left(\left|x^{\prime}\right|-L\right)^{2}+y^{\prime 2}\right)}{2 L^{2}}\right) & \text { if }\left|x^{\prime}\right|>L,\end{cases}
$$

is first introduced, after which the initial effective temperature is given by

$$
\chi(\mathbf{x}, t)=\chi_{0}+\left(800 \mathrm{~K}-\chi_{0}\right) \Gamma^{\prime}\left(x \cos 30^{\circ}+y \sin 30^{\circ},-x \sin 30^{\circ}+y \cos 30^{\circ}\right),
$$

where $\chi_{0}=600 \mathrm{~K}$. Figure 6 shows several snapshots of the effective temperature field using the quasi-static method, where the boundary conditions are set using $\zeta=10^{4}$. Shear bands nucleate from the ends of the line and grow horizontally, although they follow slightly curved paths. By $t=200 t_{s}$ the region between the two shear bands has undergone a significant increase in $\chi$.

A corresponding explicit simulation was carried out and four non-dimensionalized norms $\left\|\mathbf{v}_{\mathrm{E}}-\mathbf{v}_{\mathrm{Q}}\right\| / U_{\mathrm{B}},\left\|\boldsymbol{\sigma}_{\mathrm{E}}-\boldsymbol{\sigma}_{\mathrm{Q}}\right\| / s_{\mathrm{Y}},\left\|\chi_{\mathrm{E}}-\chi_{\mathrm{Q}}\right\| / \chi_{\infty}$, and $\left\|\boldsymbol{\xi}_{\mathrm{E}}-\boldsymbol{\xi}_{\mathrm{Q}}\right\| / L$ were evaluated at intervals $0.2 t_{s}$, where the subscripts of $\mathrm{E}$ and $\mathrm{Q}$ refer to the explicit and quasi-static simulation fields respectively. The norms provide a measure of the global differences between the fields, and the normalizing factors are chosen to make the fields in each norm approximately of order unity. Plots of the differences in these fields are shown in Fig. 7. Throughout the simulation, all fields remain in good agreement. The largest discrepancies are in the initial interval from $0 \leq t<25 t_{s}$, where all for norms exhibit 

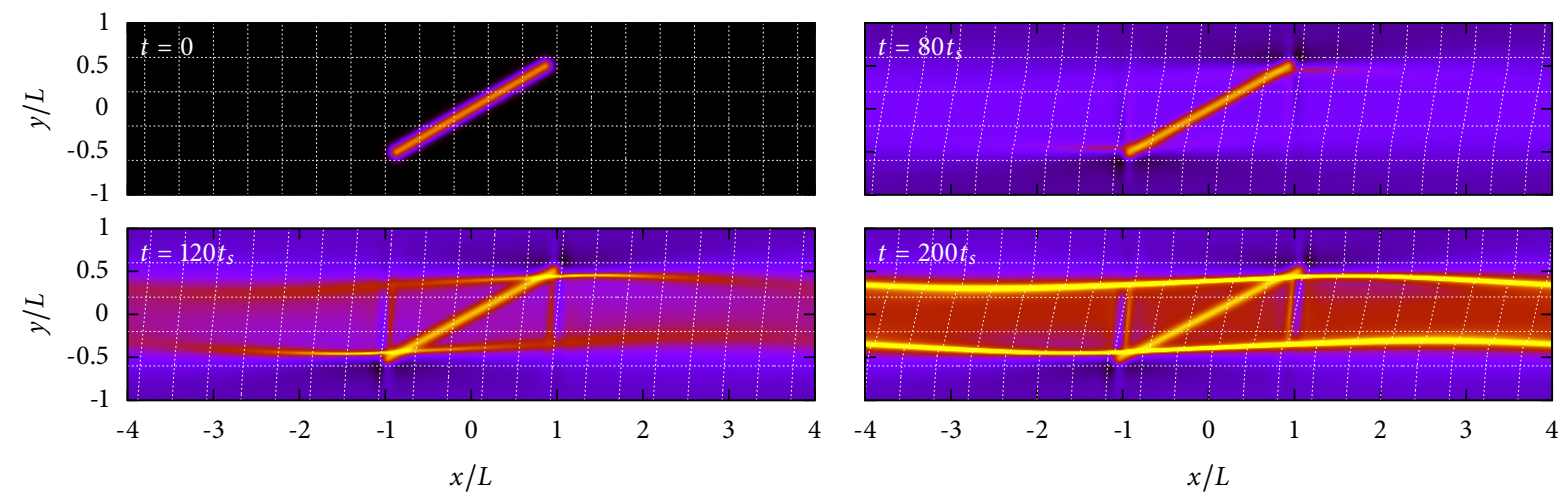

Figure 6: Four snapshots of the effective temperature $\chi$ field for a quasi-static simulation where a line of higher $\chi$ is initially introduced at an angle of $30^{\circ}$ relative to the horizontal. The simulation parameters are speeded up by a factor of $\zeta=10^{4}$ from physically realistic values. The color gradient is the same as that used in Fig. 3.

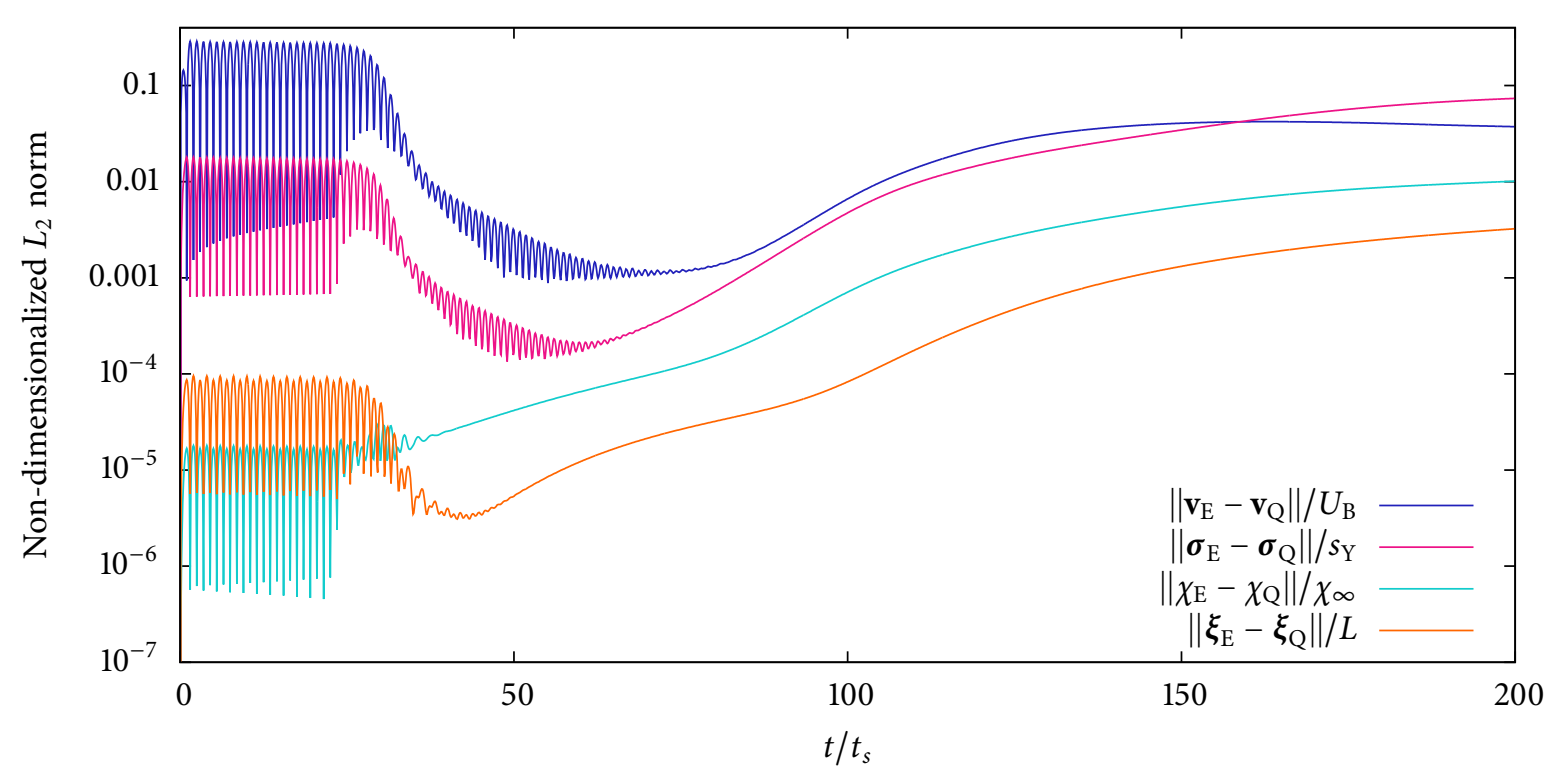

Figure 7: Non-dimensionalized differences between the simulation fields in the quasi-static and explicit simulations of the rotated line configuration shown in Fig. 6, quantified using the $L_{2}$ norm defined in the text.

oscillations. This is due to elastic waves propagating across the explicit simulation, as discussed for Fig. 5. Once plastic deformation starts to occur at $t \approx 25 t_{s}$ these oscillations are damped out, and the agreement between stresses and velocities is improved by two orders of magnitude. Beyond $t=75 t_{s}$, when the shear bands start to fully develop, all four of the norms start to increase, as small differences between the two simulations build up over time.

Figure 8 shows a comparison of the norms for the cases of $\zeta=10^{4}, 5 \times 10^{3}, 2.5 \times 10^{3}, 1.25 \times 10^{3}$. In the interval $25 t_{s}<t<75 t_{s}$ there is some limited improvement in the agreement between the methods, but for $t>75 t_{s}$, all four simulations have near-identical differences, suggesting that the dominant factor is not $\zeta$ but a difference in the discretization. Figure 9 shows several simulations for $\zeta=1.25 \times 10^{3}$, where 

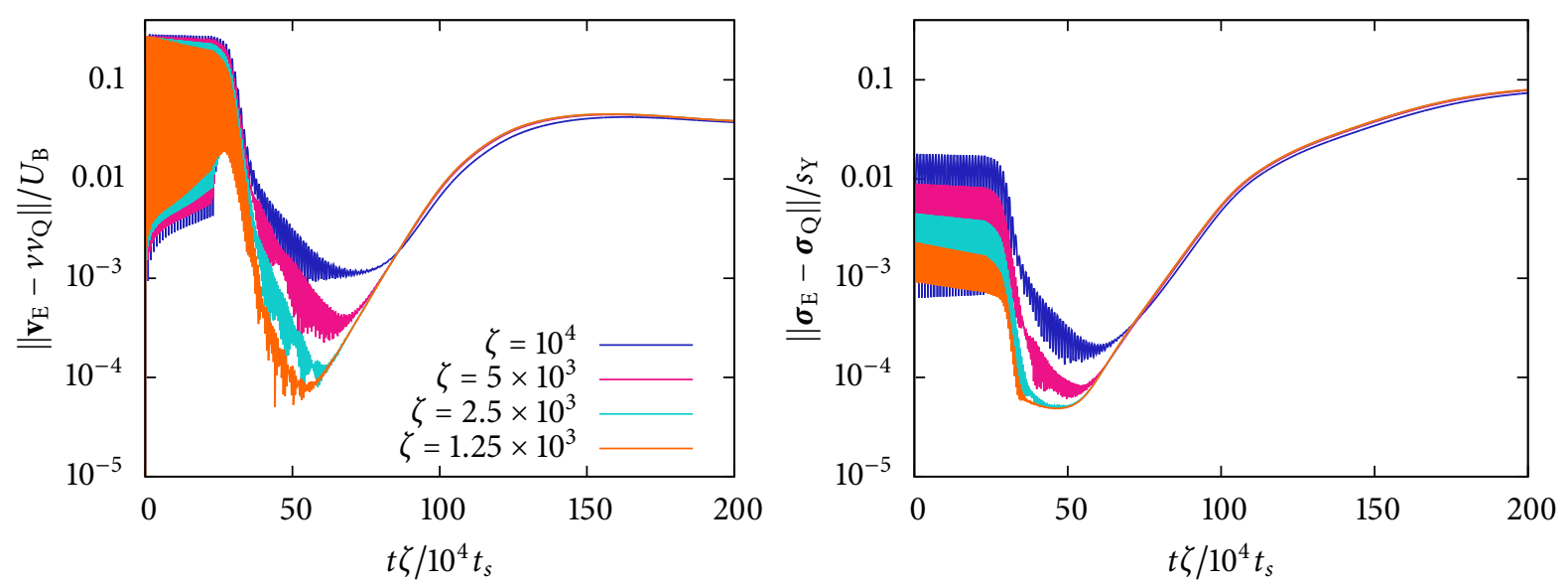

Figure 8: Non-dimensionalized differences between the velocity and stress fields in quasi-static and explicit simulations of the rotated line configuration, using four different speedup factors $\zeta$. The $L_{2}$ norm defined in the text is used.
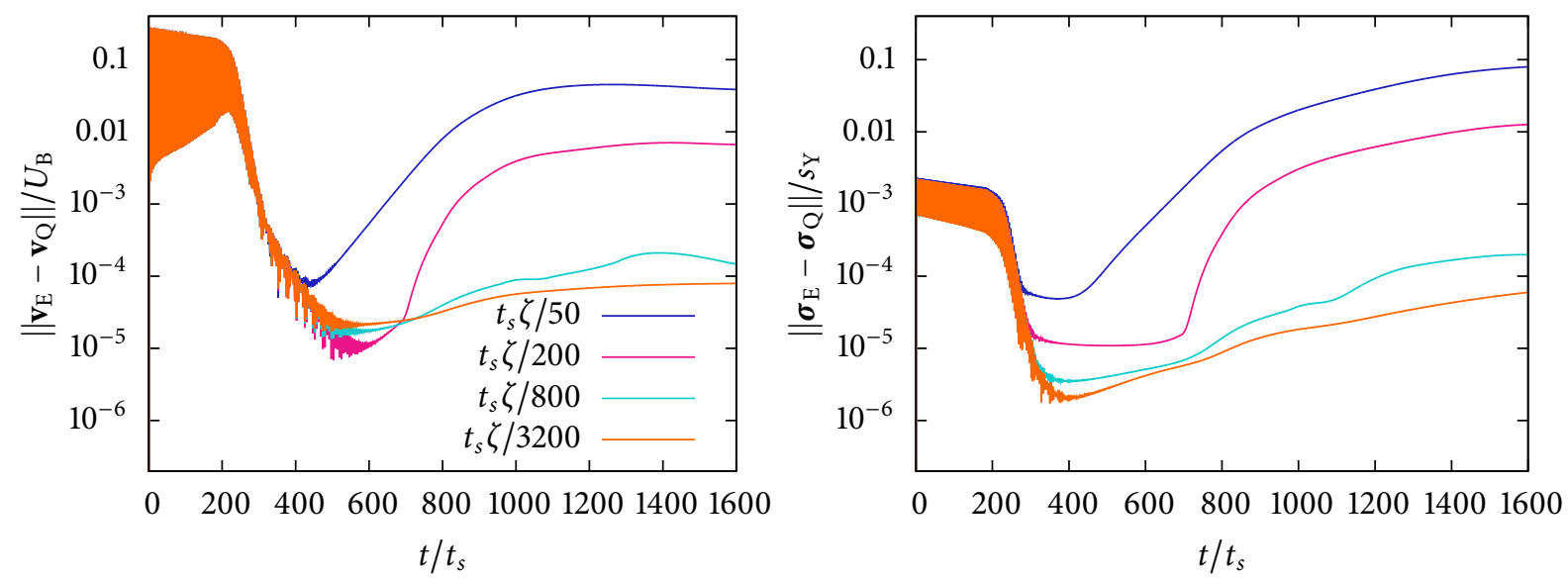

Figure 9: Non-dimensionalized differences between the velocity and stress fields in quasi-static and explicit simulations of the rotated line configuration, for four different quasi-static timestep sizes. The $L_{2}$ norm defined in the text is used.

the quasi-static timestep size is reduced by factors of four, sixteen, and 64, significantly improving the agreement for $t>75 t_{s}$. However, the agreement for the range $25 t_{s}<t<75 t_{s}$ is unchanged. Comparisons were also carried out using the original quasi-static timestep and $\zeta=10^{4}$ for two larger initial effective temperatures $\chi_{0}$ in Eq. 65. Figure 10 shows snapshots of these two simulations for $\chi_{0}=630 \mathrm{~K}$ and $\chi_{0}=660 \mathrm{~K}$ at $t=200 t_{s}$. For $\chi_{0}=630 \mathrm{~K}$, there is still some evidence of shear bands nucleating from $(x, y) \approx( \pm 0.5 L, \pm L)$, although they are much weaker than in Fig. 6 , and there is also a large diffuse band of higher effective temperature in the region $|y|<0.5 \mathrm{~L}$. For $\chi_{0}=660 \mathrm{~K}$ the thin shear bands are no longer visible, and instead the large diffuse band dominates. Figure 11 shows the differences in between the explicit and quasi-static simulations for the three different values of $\chi_{0}$. The simulations for the higher $\chi_{0}$ are in significantly closer agreement.

Taken together, Figs. 8-11 clarify the role of discretization errors in differences between the two 

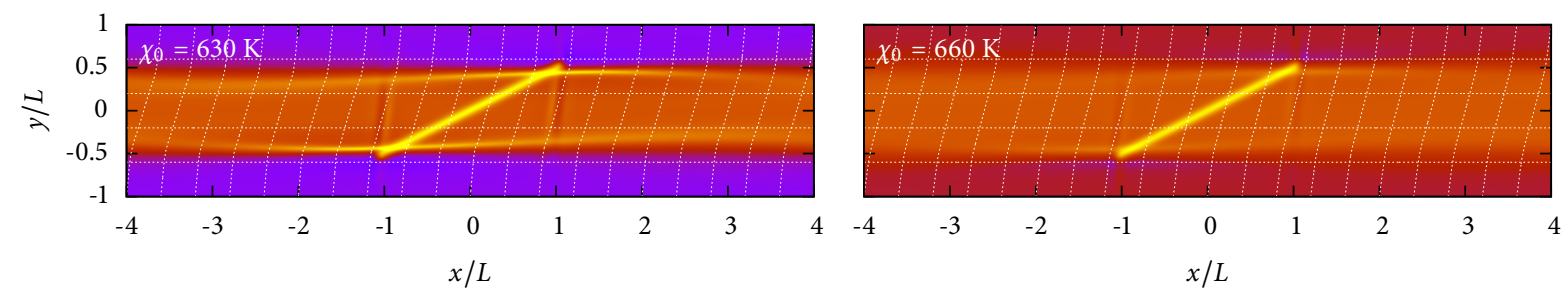

Figure 10: Quasi-static simulation snapshots at $t=200 t_{s}$ for two higher initial values of effective temperature $\chi_{0}$. The color gradient is the same as that used in Fig. 3.
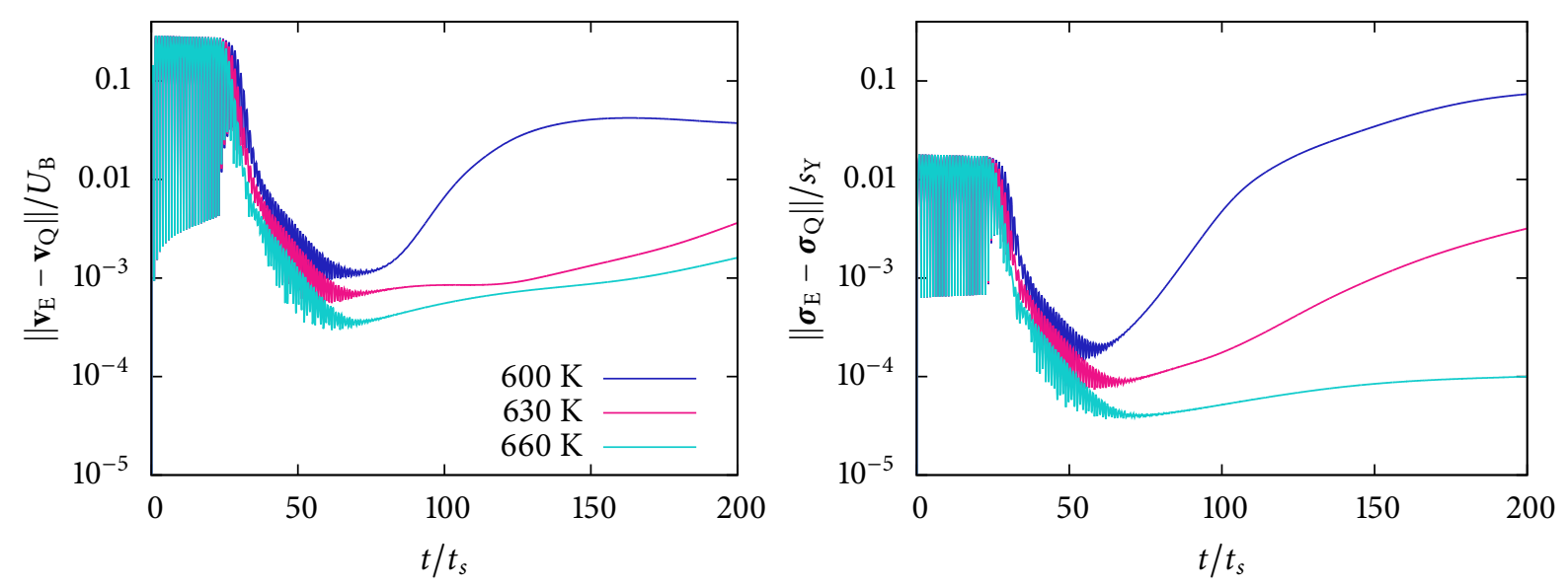

Figure 11: Non-dimensionalized differences between the velocity and stress fields in quasi-static and explicit simulations of the rotated line configuration, for three different initial background effective temperatures $\chi_{0}$. The $L_{2}$ norm defined in the text is used.

simulations. The largest differences are caused by the presence of thin shear bands. Since these features may propagate rapidly across the grid, a relatively small quasi-static timestep is required in order to properly resolve them. With these results in mind, we now return to the original question of showing an improvement in agreement between the two methods as $\zeta$ is reduced. Based on the previous results, we examine the case of $\chi_{0}=630 \mathrm{~K}$ and a quasi-static timestep of $\frac{t_{5} \zeta}{800}$, where we expect that the discretization errors between the two simulations will be small. Figure 12 shows the differences for four values of $\zeta$ and confirms that the differences are reduced for the entire duration of the simulation as $\zeta$ is lowered. For $\zeta=1.25 \times 10^{3}$, other than the initial elastic wave transients, the velocity norm remains below $10^{-4}$ and the stress norm remains below $10^{-5}$ for the entire duration of the simulation, providing confidence that the two methods are in very close agreement.

\subsection{Quasi-static simulations of physically realistic timescales}

For realistic strain rates, the explicit simulation method becomes prohibitively expensive but the quasi-static simulation method remains feasible. Here, we demonstrate this capability by simulating an example using $\zeta=1$. In the previous examples considered, there is a strong tendency for shear bands to form horizontally, even when a non-horizontal feature is present. Here, we consider a case specifically aimed at forcing a curved shear band to form. Sixteen positions 

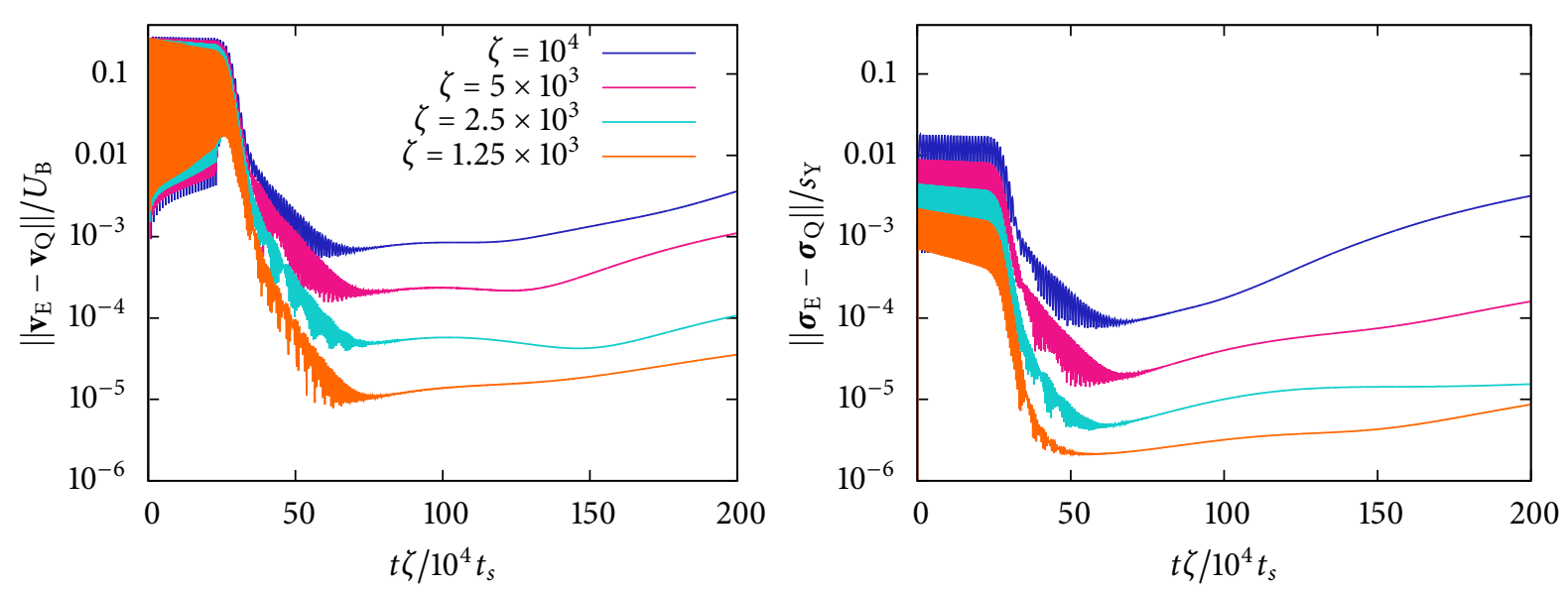

Figure 12: Non-dimensionalized differences between the velocity and stress fields in quasi-static and explicit simulations of the rotated line configuration for an initial background effective temperature of $\chi_{0}=630 \mathrm{~K}$ and a quasi-static timestep of $\frac{\zeta t_{s}}{800}$, for four different speedup factors $\zeta$. The $L_{2}$ norm defined in the text is used.

$\mathbf{x}_{k}=\left(\frac{k L}{2}+\frac{L}{2},-\frac{L}{5} \sin \left(\frac{\pi}{4}\left(\frac{k}{2}+\frac{1}{4}\right)\right)\right)$ for $k=-8,-7, \ldots, 7$ in the shape of a sine wave are introduced, and the effective temperature is initialized to be

$$
\chi(\mathbf{x}, t)=620 \mathrm{~K}+(180 \mathrm{~K}) \exp \left(-\frac{20^{2} \min _{k}\left\{\left|\mathbf{x}-\mathbf{x}_{k}\right|^{2}\right\}}{2 L^{2}}\right) .
$$

Figure 13 shows a sequence of snapshots of effective temperature and pressure. By $t=150 t_{s}$, a sinusoidal shear band has formed that links together the initial regions of higher $\chi$. However, shearing along this sinusoidal band causes material to pushed towards the region of $(x, y)=(0,4 L)$ and be pulled away from $(x, y)=(0,0)$, resulting in large positive and negative pressures respectively at these locations. By $t=300 t_{s}$, a further pair of shear bands start to emerge, which become fully developed by $t=600 t_{s}$. The additional shear bands allow the material to shear more easily and the pressure is reduced.

\section{Free boundary simulations}

The two-dimensional shearing simulations that have been considered in the previous sections employ simple boundary conditions where the velocity is prescribed on all of the physical boundaries. This leads to Dirichlet boundary conditions for the elliptic problem in the projection step, which are straightforward to implement. In this section, we extend the method to handle objects with moving boundaries to make it applicable to more general solid mechanics problems. We focus on the application of a traction-free condition $\boldsymbol{\sigma} \cdot \hat{\mathbf{n}}=\mathbf{0}$ at a boundary where $\hat{\mathbf{n}}$ is an outward-pointing normal vector.

There is again a close parallel with the fluid projection method, where conditions such as $\mathbf{v} \cdot \hat{\mathbf{n}}=0$ are frequently applied to enforce no normal flow across an impermeable boundary. At the end of a timestep, one wishes to enforce that $\hat{\mathbf{n}} \cdot \mathbf{v}_{n+1}=0$. Taking the inner product of Eq. 12 with $\hat{\mathbf{n}}$ yields

$$
\frac{\rho \hat{\mathbf{n}} \cdot \mathbf{v}_{\star}}{\Delta t}=\nabla p_{n+1}
$$




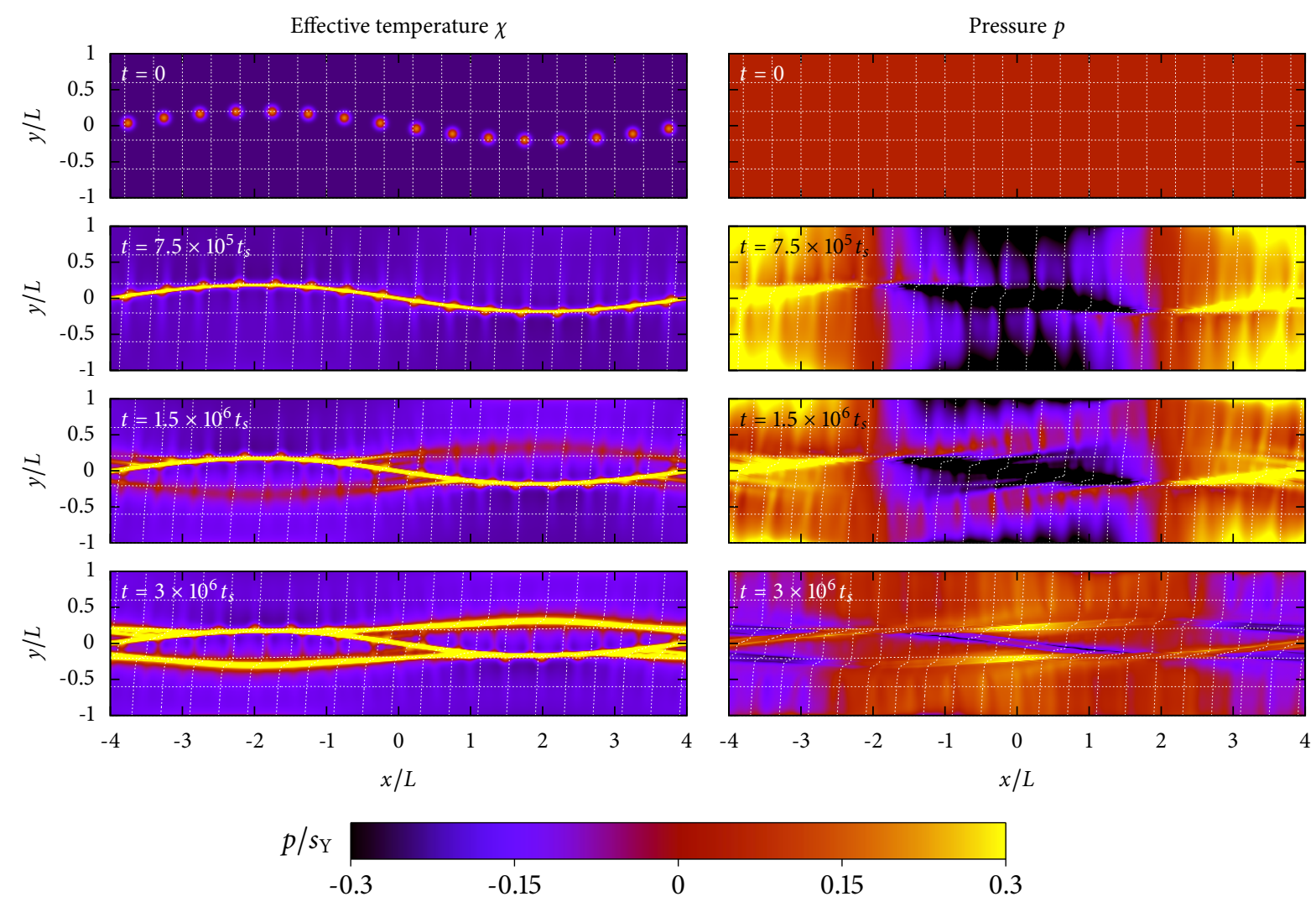

Figure 13: Snapshots of effective temperature $\chi$ and pressure $p$ for a quasi-static simulation with $\zeta=1$. The color gradient for the effective temperature is the same as that used in Fig. 3.

which is a Neumann condition in the elliptic problem for $p_{n+1}$. In a case when all boundaries in a computation are of the form $\mathbf{v} \cdot \hat{\mathbf{n}}=0$, so that the elliptic problem for pressure only employs Neumann conditions, the pressure is only determined up to an additive constant.

Analogous steps can be taken for quasi-static elastoplasticity to apply the traction-free condition at the end of a time step, so that $\hat{\mathbf{n}} \cdot \boldsymbol{\sigma}_{n+1}=\mathbf{0}$. Taking the inner product of Eq. 17 with $\hat{\mathbf{n}}$ yields

$$
-\frac{\hat{\mathbf{n}} \cdot \boldsymbol{\sigma}_{*}}{\Delta t}=\mathbf{C}: \mathbf{D}_{n+1} .
$$

This is similar to a Neumann condition: it enforces two conditions on the gradients of the components of $\mathbf{v}$, although there is also a coupling. If a problem is considered where traction-free conditions are applied everywhere, such as for an object freely floating in space, then the velocity will only be determined up to an additive vector constant. This is physically reasonable since the original system of equations, Eqs. 1 and 6, does not have any preferred velocity. Pinning the velocity at a single point in a freely floating body is enough to set the additive constant and determine the entire velocity field.

\subsection{Boundary representation}

To track the free boundary of an object we make use of the level set method [54], whereby an auxiliary function $\phi(\mathbf{x}, t)$ is introduced and is initialized to be the signed distance to the boundary, 
with the convention that $\phi(\mathbf{x}, t)<0$ inside the simulated object and $\phi(\mathbf{x}, t)>0$ outside the object. The level set method is well-suited to an Eulerian framework, since the function $\phi$ can be discretized on the same Cartesian grid as other simulation fields. It provides an implicit representation of the boundary as the zero contour, $\phi(\mathbf{x}, t)=0$. The method is widely used in fluid mechanics, since it can easily handle large stretches and topology changes in the boundary.

In principle, given an interface moving according to a globally defined velocity field $\mathbf{v}(\mathbf{x}, t)$, the function $\phi(\mathbf{x}, t)$ can be updated by using the transport equation

$$
\frac{\partial \phi}{\partial t}+(\mathbf{v} \cdot \nabla) \phi=0
$$

However in practice this causes a number of numerical difficulties: while the zero contour of $\phi$ will remain at the interface, the function $\phi$ may no longer be a signed distance function to the interface. In addition, for the current problem the simulation fields only exist on one side of the level set, inside the object where $\phi(\mathbf{x}, t) \leq 0$, and is therefore not clear what value of $\mathbf{v}$ to use in Eq. 69 .

These issues have been extensively studied over the past two decades and for a full treatment the reader should consult the books by Sethian [55] and Osher [56]. The signed distance property can be maintained by periodically reinitializing $\phi$, such as by using a PDE-based approach [67] or by a fast marching method [55]. Given fields defined inside a body, the level set function can also be used to extrapolate those fields along rays normal to the interface [68], which can be used to apply boundary conditions, or to construct a globally defined $\mathbf{v}$ in order to apply Eq. 69. For computational efficiency, the level set function only needs to be stored on a narrow band of grid points surrounding $\phi(\mathbf{x}, t)=0$.

For the examples considered here, we make use of the specific level set implementation that was previously developed for simulating elastoplastic dynamics [36]. The method employs a narrowbanded level set for efficiency, and makes use of a combination of a second-order fast marching method and the modified Newton-Raphson algorithm of Chopp [69]. It continually keeps the level set function close to a signed distance function, without the need for specific reinitialization operations. The simulation fields can be linearly extrapolated. We also make use of routines first discussed in Kamrin et al. [32] that can linearly extrapolate fields stored on a grid staggered with respect to the level set field. In the examples that follow, the results are not strongly dependent on the specifics of the level set implementation and we therefore refer the reader to these previous papers for more details.

\subsection{Numerical framework}

The examples considered here make use of a non-periodic grid of $M \times N$ square cells. As in the previous sections, the stress and effective temperature are stored at cell centers, while the velocity field and reference map are stored at cell corners. The level set field is stored at cell centers, and is initialized to represent a shape that is attached to the boundary at one or more locations, where the conditions

$$
\mathbf{v}(\mathbf{x}, t)=\mathbf{0}, \quad \boldsymbol{\xi}(\mathbf{x}, t)=\mathbf{x}
$$

are used. The simulation fields are only updated at grid points that are inside the body. A cell center $\left(i+\frac{1}{2}, j+\frac{1}{2}\right)$ is defined as inside the body if the level set field satisfies $\phi_{i+1 / 2, j+1 / 2}<0$. A cell center 
$(i, j)$ is defined as inside the body if the bilinear interpolation of the level set field

$$
\phi_{i, j}^{\prime}=\frac{\phi_{i-1 / 2, j-1 / 2}+\phi_{i+1 / 2, j-1 / 2}+\phi_{i-1 / 2, j+1 / 2}+\phi_{i+1 / 2, j+1 / 2}}{4}
$$

satisfies $\phi_{i, j}^{\prime}<0$. As described above, given a particular simulation field $f_{i, j}$ defined at grid points inside the body, linearly extrapolated values $f_{i, j}^{\mathrm{ex}}$ at points outside the body can be calculated. Prior to carrying out a simulation, all fields are extrapolated.

To carry out a timestep of $\Delta t$ in the free boundary simulations, the following procedure is used for both the explicit and quasi-static methods:

1. Move the level set according to the velocity field.

2. Using the new level set values, update which points are inside the body. Initialize the simulation fields any new grid points inside the body to be equal to the extrapolated values.

3. Calculate the finite-difference update using either the explicit method described in Subsec. 3.3 or the quasi-static method described in Subsec. 3.4, taking into account boundary conditions at the free boundary.

4. Extrapolate all fields.

5. Enforce the boundary conditions of Eq. 70 .

Step 3 requires additional consideration for both the explicit and quasi-static methods. In the explicit simulation, the velocity $\mathbf{v}$, reference map field $\xi$, and effective temperature $\chi$ are unconstrained at the free boundary. Hence, when a finite-difference calculation references any exterior point, it makes use of the available extrapolated value. The simulation only ever makes use of the exterior points that are directly adjacent to interior points. If an ENO calculation would reference a exterior point that is two points away from the interior, then the simulation falls back on a first-order upwinded derivative.

The stress tensor $\sigma$ must be handled differently in order to apply the traction-free boundary condition $\boldsymbol{\sigma} \cdot \hat{\mathbf{n}}=\mathbf{0}$. When calculating the advective derivatives, the simulation makes use of the same procedure as described in previous work [36], where a modified extrapolated value is calculated so that the linear interpolation of the stress field will satisfy the traction-free condition at the precise location of the zero level set. In addition to this, a similar procedure must be introduced to handle the boundary condition when evaluating the stresses in Eqs. 39 and 40 since the velocity field is staggered with respect to the stress field. Consider updating the velocity at a grid location $(i, j)$ and suppose that the corner $\left(i+\frac{1}{2}, j+\frac{1}{2}\right)$ is an exterior point. Then

$$
\alpha=\frac{\phi_{i, j}}{\phi_{i, j}-\phi_{i+1 / 2, j+1 / 2}}
$$

represents the position along the diagonal line from $(i, j)$ to $\left(i+\frac{1}{2}, j+\frac{1}{2}\right)$ where the zero level set intersects. At this intersected position, an interpolated stress is calculated as

$$
\boldsymbol{\sigma}_{P}=\frac{(1+\alpha) \boldsymbol{\sigma}_{i+1 / 2, j+1 / 2}+(1-\alpha) \boldsymbol{\sigma}_{i-1 / 2, j-1 / 2}}{2}
$$

and a normal vector is calculated as the gradient of the bilinear interpolation of $\phi$. Following previous work [36] a new $\boldsymbol{\sigma}_{P}^{\prime}$ is then constructed where the normal-normal and normal-tangential stress 


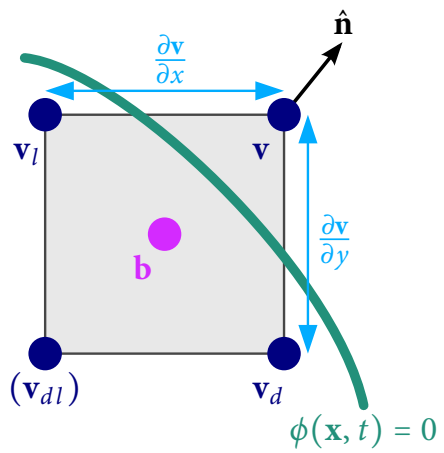

(a)

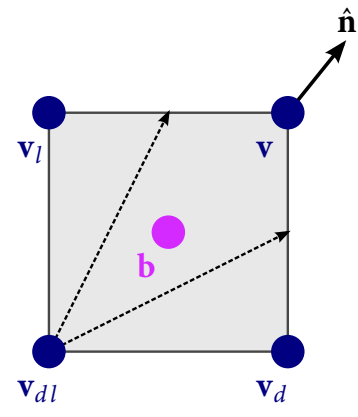

(b)

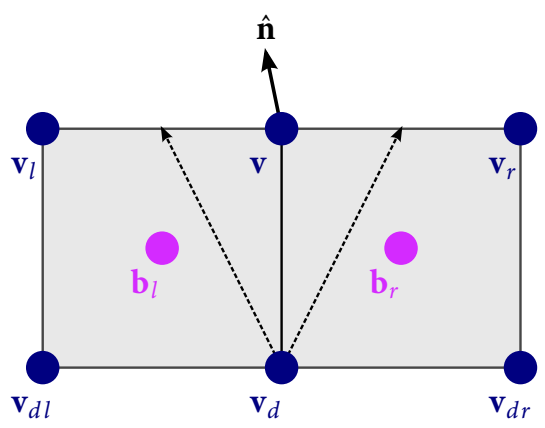

(c)

Figure 14: (a) Schematic of the basic procedure to set the velocity $\mathbf{v}$ at an exterior point to be consistent with the tractionfree boundary condition. The boundary condition involves the source term $\mathbf{b}$ at the cell center, the normal vector $\hat{\mathbf{n}}$ at the exterior point, and the derivatives $\partial_{x} \mathbf{v}$ and $\partial_{y} \mathbf{v}$, which can be approximated by $\left(\mathbf{v}-\mathbf{v}_{l}\right) / h$ and $\left(\mathbf{v}-\mathbf{v}_{d}\right) / h$ respectively. $(b, c)$ Representative diagrams showing the two types of boundary conditions, which are used if $\hat{\mathbf{n}}$ lies within the range of angles shown by the dashed arrows.

components are projected to zero. Finally, a modified extrapolated value at $\left(i+\frac{1}{2}, j+\frac{1}{2}\right)$ is calculated as

$$
\boldsymbol{\sigma}_{i+1 / 2, j+1 / 2}^{\prime}=\frac{2 \boldsymbol{\sigma}_{i-1 / 2, j-1 / 2}-(1-\alpha) \boldsymbol{\sigma}_{P}^{\prime}}{1+\alpha},
$$

which is then used in the finite-difference calculation of Eqs. 39 and 40.

\subsection{Boundary implementation in the projection step}

The projection step in the quasi-static method must also be modified to take into account the free boundary. The velocity fields must only be solved at grid points within the body. At these points, the linear system is constructed in the same manner as previously, using the discretization of Eqs. 57 and 58. The discretization will also reference exterior grid points that are either orthogonally or diagonally adjacent to an interior point-we refer to this set of outside points as neighboring points.

At the neighboring points, we also solve for the velocity in the linear system, and calculate values that are consistent with the boundary condition in Eq. 68, which is

$$
\begin{gathered}
\frac{1}{\Delta t} \hat{\mathbf{n}} \cdot\left(\begin{array}{cc}
-p_{*}-q_{*}+s_{*} & \tau_{*} \\
\tau_{*} & -p_{*}-q_{*}-s_{*}
\end{array}\right) \\
=\hat{\mathbf{n}} \cdot\left(\begin{array}{cc}
-K^{\prime}\left(u_{x}+v_{y}\right)-\mu\left(u_{x}-v_{y}\right) & -\mu\left(u_{y}+v_{x}\right) \\
-\mu\left(u_{y}+v_{x}\right) & -K^{\prime}\left(u_{x}+v_{y}\right)+\mu\left(u_{x}-v_{y}\right)
\end{array}\right)
\end{gathered}
$$

when expressed in terms of the simulation fields. Applying this condition is similar to extrapolation $[68,55,36]$, in that the velocities at the neighboring points are normally extended from the interior points in a manner that satisfies Eq. 75.

To illustrate this procedure, consider the basic example shown in Fig. 14(a), where the velocity at the neighboring point $\mathbf{v}$ can be expressed in terms of the velocities at the interior points $\mathbf{v}_{d}$ and $\mathbf{v}_{l}$. 
One-sided first derivatives of $\mathbf{v}$ are given by

$$
\frac{\partial \mathbf{v}}{\partial x}=\frac{\mathbf{v}-\mathbf{v}_{l}}{h}, \quad \frac{\partial \mathbf{v}}{\partial y}=\frac{\mathbf{v}-\mathbf{v}_{d}}{h} .
$$

A normal vector $\hat{\mathbf{n}}$ is calculated at $\mathbf{v}$. A source term $\mathbf{b}=-\frac{\hat{\mathbf{n}} \cdot \boldsymbol{\sigma}_{\star}}{\Delta t}$ is then calculated at the center of the square. If the two matrices

$$
H(\hat{\mathbf{n}})=\frac{1}{h}\left(\begin{array}{cc}
-(K+\mu) n_{x} & -\mu n_{y} \\
(\mu-K) n_{y} & -\mu n_{x}
\end{array}\right), \quad V(\hat{\mathbf{n}})=\frac{1}{h}\left(\begin{array}{cc}
-\mu n_{y} & (\mu-K) n_{x} \\
-\mu n_{x} & -(K+\mu) n_{y}
\end{array}\right)
$$

are introduced, then Eq. 75 can be implemented as

$$
H(\hat{\mathbf{n}})\left(\mathbf{v}-\mathbf{v}_{l}\right)+V(\hat{\mathbf{n}})\left(\mathbf{v}-\mathbf{v}_{d}\right)=\mathbf{b} .
$$

From Fig. 14(a) it can be seen that there is some freedom in choosing the precise formula for $\mathbf{v}$. For example, the the $x$-derivative could be also obtained using $\partial \mathbf{v} / \partial x=\left(\mathbf{v}_{d}-\mathbf{v}_{d l}\right) / h$. In our numerical tests, we found that the best results were achieved when extension formulae made use of a combination of the available velocities that closely matched with the direction of the normal vector. We therefore made use of two different types of numerical stencils depending on whether the normal vector pointed diagonally or orthogonally. The stencils are chosen in such a way that their values change continuously as the angle of the normal vector is varied.

The first stencil type is shown in Fig. 14(b) and is illustrated for the case when the normal vector points diagonally up-right so that $2 \hat{n}_{x}>\hat{n}_{y}$ and $2 \hat{n}_{y}>\hat{n}_{x}$. A parameter $\beta$ is defined as

$$
\beta= \begin{cases}\frac{\hat{n}_{x}}{2 \hat{n}_{y}} & \text { if } \hat{n}_{x}>\hat{n}_{y}, \\ 1-\frac{\hat{n}_{y}}{2 \hat{n}_{x}} & \text { if } \hat{n}_{x} \geq \hat{n}_{y}\end{cases}
$$

so that it continuously varies from 0 to 1 over the range of normal vectors considered. If $\alpha=1-\beta$, then the boundary condition is implemented as

$$
\begin{aligned}
& H(\hat{\mathbf{n}})\left[\beta\left(\mathbf{v}-\mathbf{v}_{l}\right)+\alpha\right.\left.\left(\mathbf{v}_{d}-\mathbf{v}_{d l}\right)\right]+V(\hat{\mathbf{n}})\left[\alpha\left(\mathbf{v}-\mathbf{v}_{d}\right)+\beta\left(\mathbf{v}_{l}-\mathbf{v}_{d l}\right)\right] \\
&+8 \beta \alpha(\alpha V(\hat{\mathbf{n}})+\beta H(\hat{\mathbf{n}}))\left(\mathbf{v}+\mathbf{v}_{d l}-\mathbf{v}_{l}-\mathbf{v}_{d}\right)=\mathbf{b},
\end{aligned}
$$

where the source term $\mathbf{b}$ is calculated at the center of the grid cell. This formulation therefore smoothly transitions from calculating derivatives on the bottom and right cell edges when $\beta=0$, to calculating derivatives on top and left cell edges when $\beta=1$. The third term on the left hand side of the equation amplifies the diagonal terms when the normal is close to the diagonal.

The second stencil type is shown in Fig. 14(c) and is illustrated for cases where the normal vector points upward, so that $\hat{n}_{y} \geq 2\left|\hat{n}_{x}\right|$. In this case, the parameter is given by

$$
\beta=\frac{1}{2}+\frac{\hat{n}_{x}}{\hat{n}_{y}}
$$


so that it varies from 0 to 1 over the range of normal vectors considered. If $\alpha=1-\beta$, the boundary condition is implemented as

$$
V(\hat{\mathbf{n}})\left[\mathbf{v}-\mathbf{v}_{d}\right]+H(\hat{\mathbf{n}})\left[\beta\left(\mathbf{v}_{d r}-\mathbf{v}_{d}\right)+\alpha\left(\mathbf{v}_{d}-\mathbf{v}_{d l}\right)\right]=\beta \mathbf{b}_{r}+\alpha \mathbf{b}_{l},
$$

where $\mathbf{b}_{l}$ and $\mathbf{b}_{r}$ are the source terms on the left and right grid cells. By applying flips in the $x$ and $y$ axes, the two stencils shown in Figs. 14(b) and 14(c) can be extended to handle all other directions of normal vector. As the normal vector changes, the stencil entries and the source terms that are used all vary continuously, and there are no discontinuous jumps between the different cases.

\subsection{Quasi-static loading and unloading of a bar}

The first free boundary example makes use of a horizontal bar where the right end is fixed to a wall. At the left end of the bar, a load is incrementally applied on a quasi-static timescale, and is then incrementally removed. The load is applied in a diagonal direction so that the bar is both stretched and deformed downward, and the magnitude of the load is large enough to cause a substantial amount of plastic deformation around the loading region. This leads to a complex deformation of the bar, which makes for a good numerical test of the method. By using the reference map field $\boldsymbol{\xi}(\mathbf{x}, t)$, we also demonstrate the calculation of strain in a fully Eulerian simulation, and we examine the interplay between deviatoric and volumetric strain.

The example uses the domain $-2 L \leq x \leq 2 L,-L \leq y \leq L$ with a $512 \times 256$ grid. The level set is initialized to represent a horizontal bar in the region $x>-1.65 L,|y|<0.65 L$ with rounded corners of radius $0.3 L$, due to the difficulties of accurately representing sharp corners using the level set method. The bar is fixed to the boundary at $x=2 L$, and the initial effective temperature in the bar is $620 \mathrm{~K}$. The simulation lasts for $10^{6} t_{s}$, which is $4.05 \mathrm{~s}$ for the nominal length scale of $L=1 \mathrm{~cm}$. Quasistatic timesteps of size $1250 t_{s}$ are used. The load position is given by $\mathbf{x}_{F}(t)$ with initial condition $\mathbf{x}_{F}(0)=(-L, 0)$. The load moves with the body according to

$$
\frac{d \mathbf{x}_{F}}{d t}=\mathbf{v}\left(\mathbf{x}_{F}, t\right)
$$

This equation is implemented using the Euler timestep, and the term $\mathbf{v}\left(\mathbf{x}_{F}, t\right)$ is calculated using bicubic interpolation of the velocity field. The load is applied as a body force $\mathbf{F}(\mathbf{x}, t)$ in the projection step, as an additional source term on the right hand side of Eqs. 57 and 58. The time dependence of the applied load is given by the function

$$
F_{T}(t)= \begin{cases}\frac{t}{t_{s}} & \text { for } 0 \leq t<4 \times 10^{5} t_{s} \\ 8 \times 10^{5}-\frac{t}{t_{s}} & \text { for } 4 \times 10^{5} t_{s} \leq t<8 \times 10^{5} t_{s}, \\ 0 & \text { for } 8 \times 10^{5} t_{s} \leq t \leq 10^{6} t_{s}\end{cases}
$$

so that the bar is incrementally loaded up to $t=4 \times 10^{5} t_{s}$ and then incrementally unloaded up to $t=8 \times 10^{5} t_{s}$. The spatial dependence of the applied load is given by

$$
F_{R}(r)= \begin{cases}1+\cos \frac{\pi r}{r_{F}} & \text { for } r<r_{F} \\ 0 & \text { for } r \geq r_{F}\end{cases}
$$


so that it is applied over a circle of radius $r_{F}=0.25 \mathrm{~L}$. The force is then given in terms of these two functions as

$$
\mathbf{F}(\mathbf{x}, t)=-\left(\begin{array}{c}
12 \psi \\
\psi
\end{array}\right) F_{R}\left(\left|\mathbf{x}-\mathbf{x}_{F}\right|\right) F_{T}(t)
$$

where $\psi=4.625 \times 10^{-6} s_{Y} / L$.

Figure 15 shows snapshots of the pressure and deviatoric stress in the simulation. As the bar is loaded up to $t=4 \times 10^{5} t_{s}$, negative pressures build up in the bar, apart from a small region to the left of the applied load, where a positive pressure emerges. By $t=4 \times 10^{5} t_{s}$ the deviatoric stress has exceeded $s_{\mathrm{Y}}$ in some areas, leading to plastic deformation. After the bar has been unloaded at $t=8 \times 10^{5} t_{s}$, some residual pressure and shear stress is visible as a result of the plastic deformation. While not shown, the simulation fields remain static over the interval $8 \times 10^{5} t_{s}<t \leq 10^{6} t_{s}$.

The top two plots in Fig. 16 show the effective temperature at the time of maximum load, and at the time when the load is removed. As would be expected from the regions of high deviatoric stress at $t=4 \times 10^{5} t_{s}$, regions of increased $\chi$ are visible around the loading region, and also at the top right corner, where a small shear band forms. While the bulk of the increased $\chi$ occurs during the period of increasing load, a small increase in $\chi$ is also visible during the period of decreasing load-this is expected since the plastic deformation will not immediately cease when the load starts to decrease.

Figure 16 also shows plots of the deviatoric strain measured in terms of the $\left|\mathbf{E}_{0}\right|$, and the volume ratio $\operatorname{det} \mathbf{F}$, which are computed using the reference map field $\boldsymbol{\xi}(\mathbf{x}, t)$. We use $\operatorname{det} \mathbf{F}-1$ to measure the volumetric strain. As expected, there is a high correlation between the deviatoric strain and the regions of higher $\chi$, since $\chi$ increases in regions where the material has yielded plastically, and the plastic deformation only has a deviatoric component. At the point of maximum load, the correlation is moderately high, since $\left|\mathbf{E}_{0}\right|$ will be a combination of both plastic strain, and elastic strain due to the stresses. Once the load is removed, the correlation is very high, since the $\left|\mathbf{E}_{0}\right|$ is almost entirely determined in terms of plastic strain. At both timepoints, the volumetric strain is closely correlated with pressure, since there is no volumetric plastic strain. The volumetric strain at $t=8 \times 10^{5} t_{s}$ is due to the residual pressure in the bar.

Since the majority of the load is applied horizontally, the amount that the bar stretches can be compared to an analytic estimate based on a uniaxial extension test. Let $\Omega$ be the region where the load is applied. The total horizontal force per unit length is

$$
\begin{aligned}
\bar{F}_{x}(t) & =\int_{\Omega} 12 \psi F_{R}\left(\left|\mathbf{x}-\mathbf{x}_{F}\right|\right) F_{T}(t) d^{2} \mathbf{x}=12 \psi F_{T}(t) 2 \pi \int_{0}^{r_{F}} F_{R}(r) r d r \\
& =24 \psi \pi\left(\frac{r_{F}^{2}\left(\pi^{2}-4\right)}{2 \pi^{2}}\right) F_{T}(t)=\frac{12 \psi r_{F}^{2}\left(\pi^{2}-4\right)}{\pi} F_{T}(t) \\
& =6.48 \times 10^{-6} F_{T}(t) s_{Y} L=55.1 F_{T}(t) \mathrm{N} / \mathrm{m}
\end{aligned}
$$

and hence the maximum load at $t=4 \times 10^{5} t_{s}$ is $2.59 s_{\mathrm{Y}} L$ or $22.0 \mathrm{MN} / \mathrm{m}$.

In the plane strain configuration, the effective Young's modulus is given by $E^{\prime}=E /\left(1-v^{2}\right)$. The loading point $\mathbf{x}_{F}(t)$ is initially $3 L$ from the fixed wall and the bar has width $1.3 L$. Hence the expected extension as a function of time is

$$
\Delta x_{F}(t)=\frac{\bar{F}_{x}(t) 3 L}{1.3 L E^{\prime}}=F_{T}(t) 1.10 \times 10^{-7} L .
$$




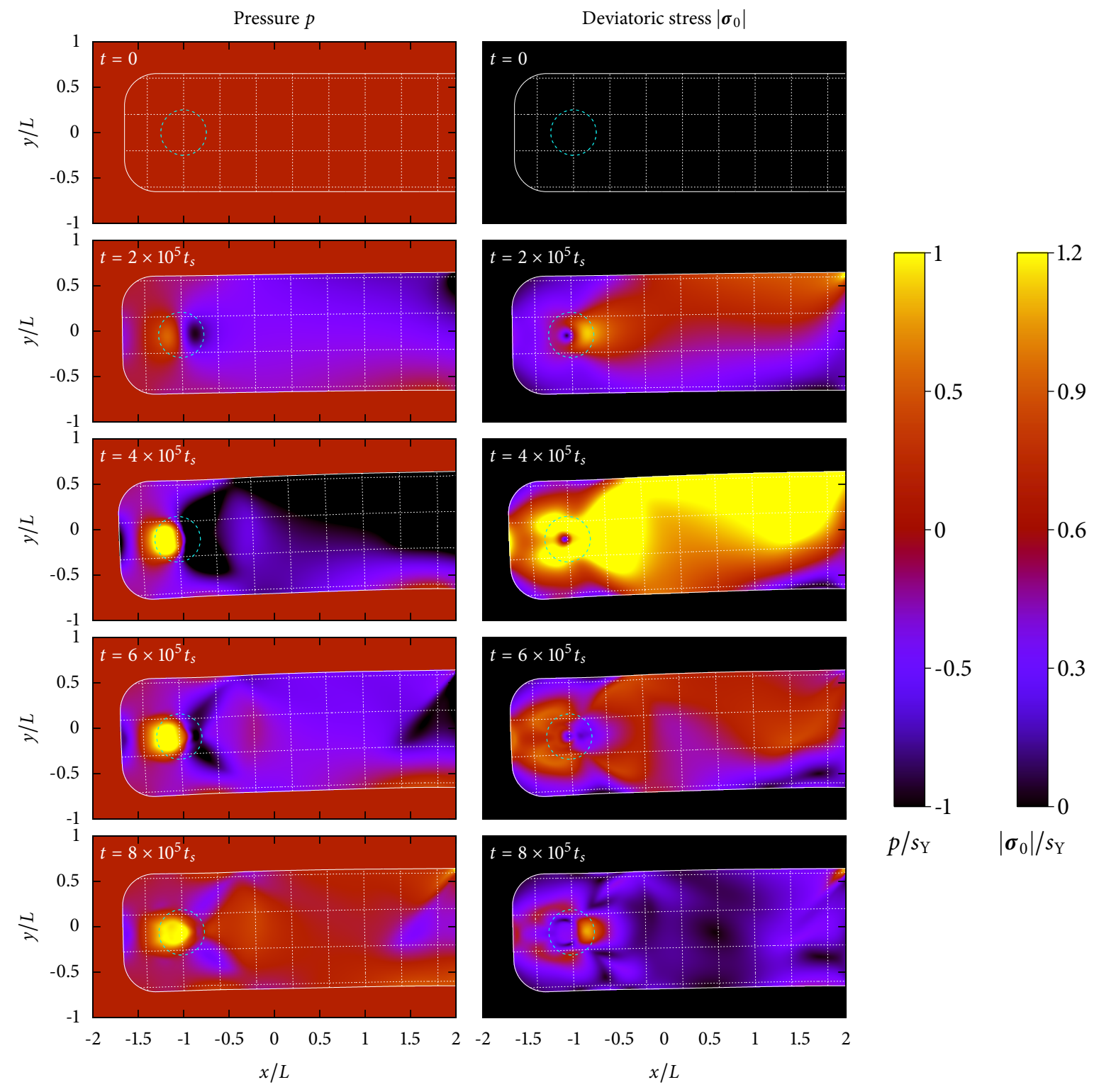

Figure 15: Plots of pressure $p$ (left) and deviatoric stress $\left|\boldsymbol{\sigma}_{0}\right|$ (right) at five time points of the stretched bar simulation. The boundary of the bar is shown as the solid white line obtained as the zero contour of level set function $\phi$. The thin dashed white lines are the contours of the components of the reference map $\xi$ and show how the material is deformed. The dashed cyan circle shows the region where the bar is loaded. 

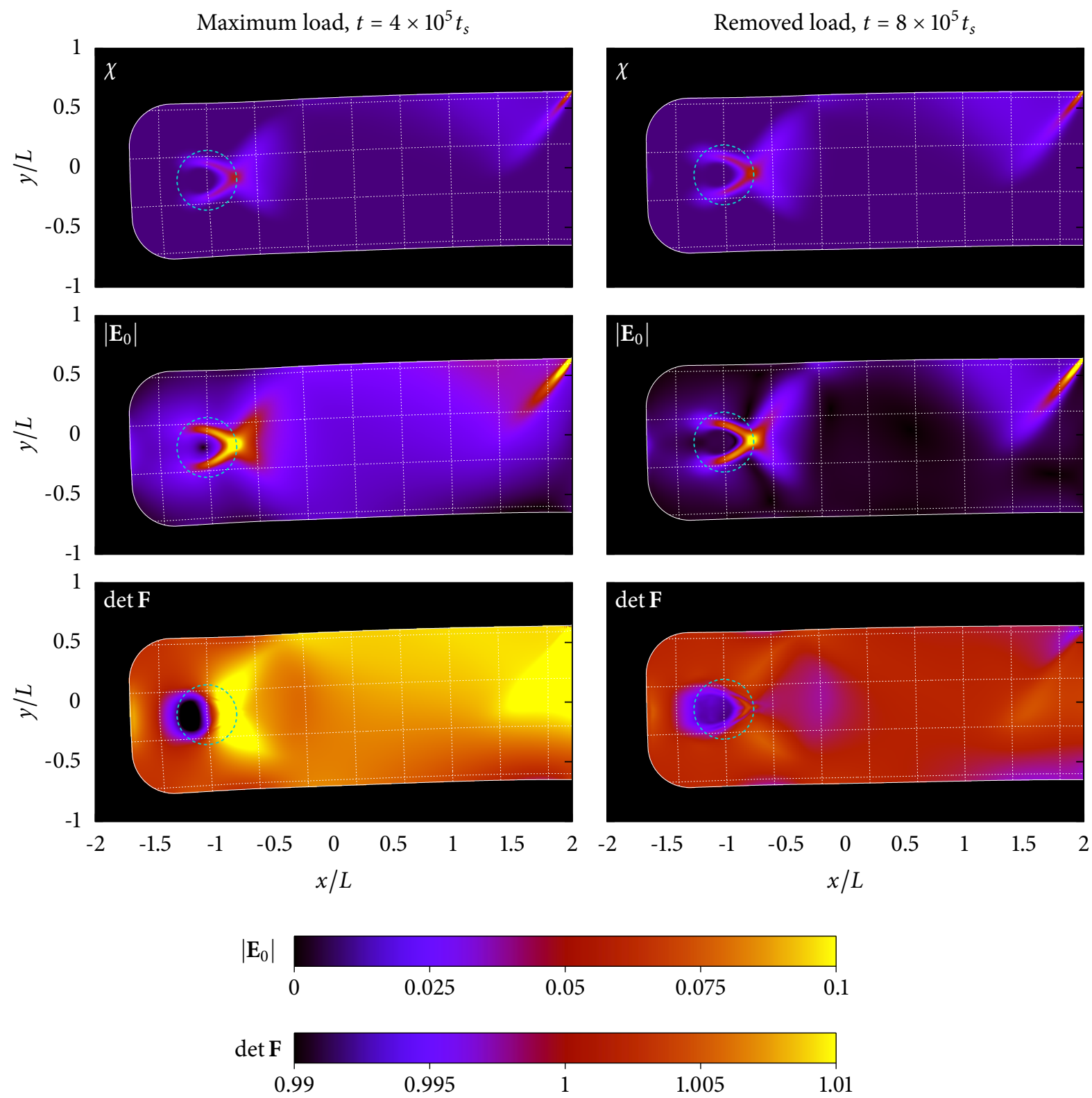

Figure 16: Plots of effective temperature $\chi$ (top), deviatoric strain $\left|\mathbf{E}_{\mathbf{0}}\right|$ (middle), and volume ratio $\operatorname{det} \mathbf{F}$ (bottom) in the stretched bar simulation at the time of maximum load (left) and at the time when the load has been removed (right). The boundary of the bar is shown as the solid white line obtained as the zero contour of level set function $\phi$. The thin dashed white lines are the contours of the components of the reference map $\xi$ and show how the material is deformed. The dashed cyan circle shows the region where the bar is loaded. The effective temperature plots use the same scale as Fig. 3, and scales for strain plots are shown. 


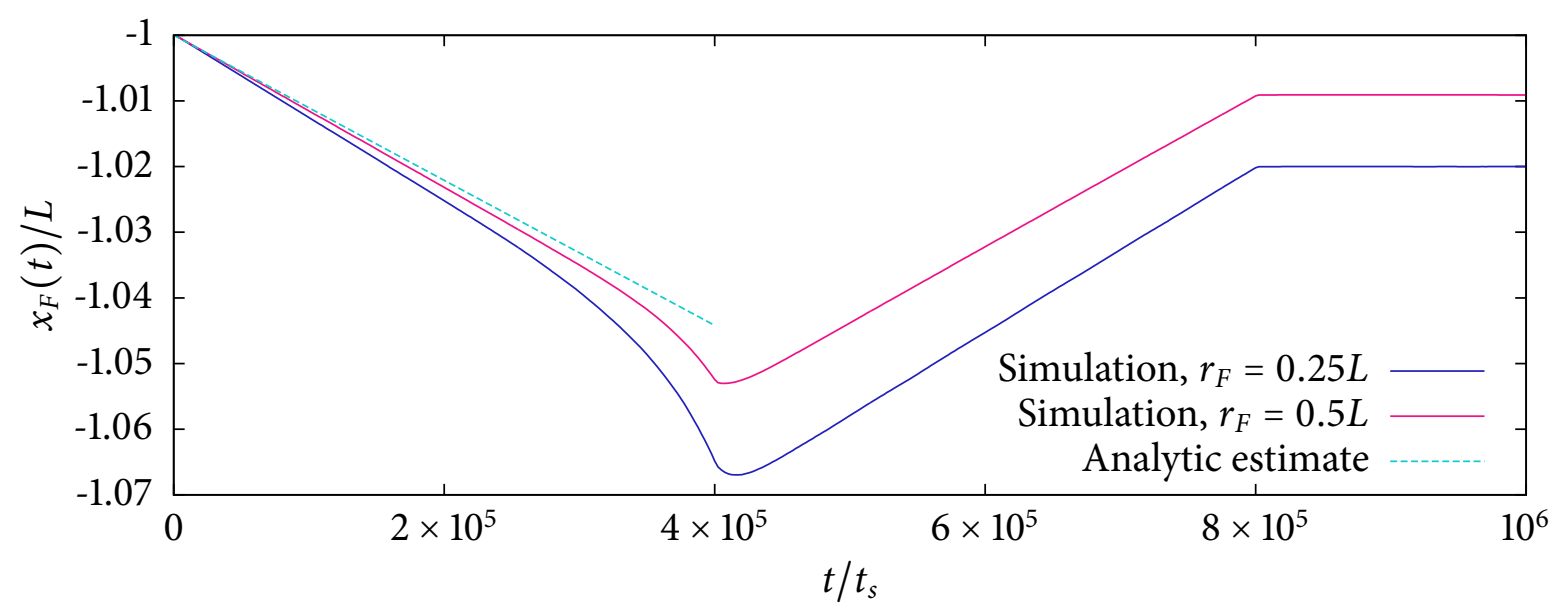

Figure 17: Time evolution of the horizontal loading position $x_{F}(t)$ for two stretched bar simulations, compared to an analytic estimate based on a uniaxial tension test.

Figure 17 shows a plot of the horizontal loading position over time in the simulation, compared to this analytic estimate. The two curves are in reasonable agreement, although the gradient of the curve close to $t=0$ has a slightly larger magnitude in the simulation. This is expected, since in the simulation the load is localized in a small central region of the bar, rather than being spread across the whole bar. This is confirmed by the plots $\left|\sigma_{0}\right|$ in Fig. 17, which show relatively low levels of stress at the edges of the bar over the range $-L<x<-0.5 L$. To confirm that this is the source of the discrepancy, a second simulation was carried out where the diameter of the loading region was doubled to $r_{F}=0.5 \mathrm{~L}$ while keeping the total load the same. As expected, the extension in this simulation is in closer agreement with the analytic estimate.

The plastic deformation of the bar is also evident in Fig. 17. As $t$ approaches $4 \times 10^{5} t_{s}$, the rate extension of the loading point noticeably increases. After the load is removed at $t=8 \times 10^{5} t_{s}$, the loading point does not fully return to its original position. Both simulation curves show the same trends although less plastic deformation is evident in the curve for $r_{F}=0.5 \mathrm{~L}$, since by spreading out the load, and hence stress, smaller regions of the bar will deform plastically.

\subsection{Transition from the quasi-static simulation to the explicit simulation}

Since the explicit and quasi-static timestepping methods make use of the same grids and fields, they can be intermixed, making it possible to simulate processes with disparate time scales. In a recent paper [57], we considered one such situation of dynamic crack propagation, where a bulk metallic glass was loaded on a time scale of seconds and first accumulates rather slow plastic deformation, but then fractures on a time scale of nanoseconds. Here, we consider another case, where a bar is loaded on a quasi-static timescale and then the load is instantaneously released, making the bar rapidly oscillate. The simulation domain is $|x| \leq 0.5 L,|y| \leq L$ using a $512 \times 1024$ grid. The level set function is initialized to be a vertical bar in the region $|x|<0.25 \mathrm{~L}$ with four holes of radius $0.15 \mathrm{~L}$ at $x= \pm 0.8 L, \pm 0.4 L$ and $y=0$. The bar is attached to the top and bottom boundaries, and the initial effective temperature is $620 \mathrm{~K}$. The loading position $\mathbf{x}_{F}$ is initially located at the origin. The temporal 
and spatial dependencies of the force are given by

$$
F_{T}(t)=\left\{\begin{array}{ll}
\frac{t}{t_{s}} & \text { for } 0 \leq t \leq t_{\mathrm{R}}, \\
0 & \text { for } 5 \times 10^{5}<t_{s} \leq t_{\mathrm{R}}+10 t_{s},
\end{array} \quad F_{R}(r)= \begin{cases}1+\cos \frac{\pi r}{r_{F}} & \text { for } r<r_{F}, \\
0 & \text { for } r \geq r_{F},\end{cases}\right.
$$

where $t_{\mathrm{R}}=5 \times 10^{5} t_{s}$ and $r_{F}=0.15 \mathrm{~L}$. The total force is then given by

$$
\mathbf{F}(\mathbf{x}, t)=\left(\begin{array}{c}
-\psi F_{R}\left(\left|\mathbf{x}-\mathbf{x}_{F}\right|\right) F_{T}(t) \\
0
\end{array}\right),
$$

where $\psi=5 \times 10^{-6} s_{\mathrm{Y}} / L$. The simulation first uses quasi-static timesteps of size $625 t_{s}$ to simulate the time interval $0 \leq t \leq t_{\mathrm{R}}$. At $t=t_{\mathrm{R}}$, the load reaches its maximum value of $0.105 s_{\mathrm{Y}} / L$, which is $0.893 \mathrm{MN} / \mathrm{m}$ in the nominal physical units. When the load is removed, the simulation switches over to explicit timesteps to simulate up to $t=t_{\mathrm{R}}+10 t_{s}$.

Figures 18 and 19 show snapshots of the pressure and deviatoric stress respectively for this simulation. In both figures, the top row shows snapshots during the quasi-static loading process. As expected, in the middle of the bar, negative pressures grow on the left of the bar as it is stretched, and positive pressures grow on the right of the bar as it is compressed. The largest deviatoric stresses develop in the small regions between each pair of holes at $\mathbf{x}=\left(0, \pm \frac{L}{2}\right)$, and also at edges of the bar close to the top and bottom boundaries. In both of these regions, $\left|\sigma_{0}\right|$ exceeds $s_{\mathrm{Y}}$ and hence plastic deformation takes place.

In Figs. 18 and 19 the bottom row of snapshots show the bar at several points after the load has been released. As soon as the load is released, elastic waves rapidly propagate through the bar, and the stress imbalance pushes the bar rightward. Figs. 18(d) and 19(d) show the bar when it first reaches an approximately vertical state. Some small concentrations of pressures and deviatoric stress are visible in the regions that underwent plastic deformation. In Fig. 18(e) and 19(e), the bar is shown at its maximal rightward extent. Very large deviatoric stresses are visible in the regions between each pair of holes. After this timepoint, the bar begins to move leftward. Fig. 18(f) and 19(f) show the bar when it becomes vertical for the second time.

Figure 20 shows the effective temperature in this simulation at three time points. At $t=t_{\mathrm{R}}$, as expected, an increase effective temperature is visible in the regions between the holes, and near the top and bottom boundaries. At $t=t_{\mathrm{R}}+10 t_{s}$, after the bar has undergone the rapid oscillatory motion, further increases in $\chi$ are visible in the regions between the holes. Because the oscillatary motion creates large deviatoric stresses up to $1.9 s_{Y}$, and the plasticity model specified in Eqs. 31 and 34 has an exponential dependence on $\left|\boldsymbol{\sigma}_{0}\right|$, noticeable plastic deformation can occur on a very short timescale. This is a consequence of the simplified choice of the plasticity model discussed in Subsec. 3.2.

The loading phase and release phase differ by more than four orders of magnitude in duration, and this example therefore highlights the ability to simulate phenomena across a wide range of timescales. It may also be possible to carry out an opposite transition from an explicit simulation to a quasi-static simulation, although this would require careful consideration of any elastic waves in the explicit simulation, which would immediately disappear after a single quasi-static projection step. In the free boundary examples presented here and in the previous subsection, it has been possible to determine a priori whether the quasi-static method or the explicit method should be applied, but this may not be the case in general, particularly since in an elastoplastic material the relevant 

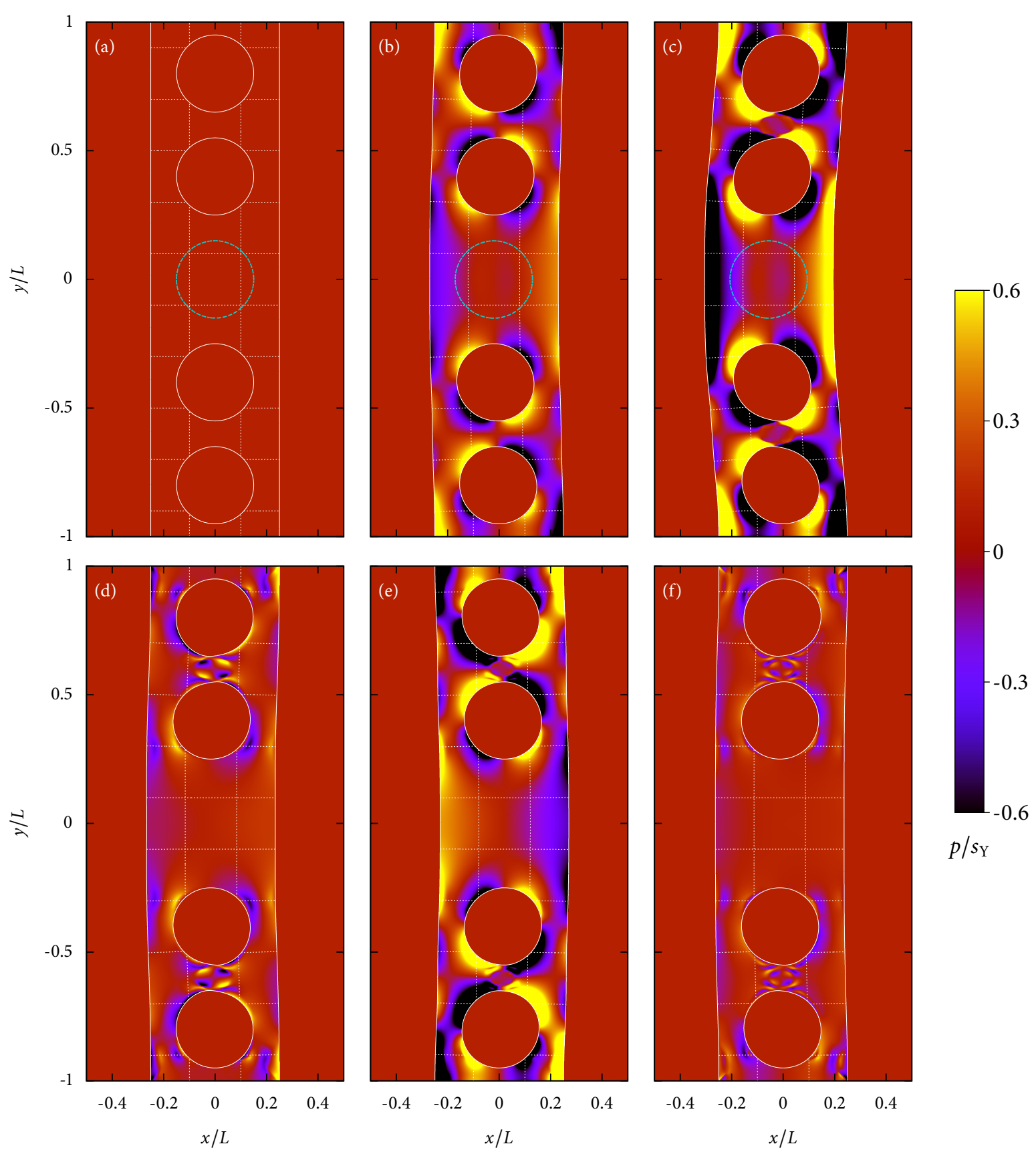

Figure 18: Plots of pressure $p$ for the load-release simulation. The top three snapshots are of the loading process simulated with the quasi-static method, at times of (a) $t=0$, (b) $t=2.5 \times 10^{5} t_{s}$, and (c) $t=t_{\mathrm{R}}=5 \times 10^{5} t_{s}$. The bottom three snapshots show the dynamics of the bar after the load is instaneously removed, simulated with the explicit method, at times of (d) $t=t_{\mathrm{R}}+2.5 t_{s}$, (e) $t=t_{\mathrm{R}}+5 t_{s}$, and (f) $t=t_{\mathrm{R}}+7.5 t_{s}$. The boundary of the bar is shown as the solid white line obtained as the zero contour of level set function $\phi$. The thin dashed white lines are the contours of the components of the reference map $\xi$ and show how the material is deformed. For plots (a) to (c), the dashed cyan circle shows the region where the bar is loaded. 
timescales may dynamically change. In Fig. 17, the loading position starts to move more quickly near the time of maximum load, due to the positive feedback between effective temperature and $\mathbf{D}^{\mathrm{pl}}$, and for larger loads, the motion may become so great that quasi-staticity may no longer be a reasonable assumption. We expect that this can be quantified by examining the size of the projection required to restore quasi-staticity, creating the possibility of automatically selecting the correct time-integration method to use, although we leave this for the subject of future work.

\section{Conclusion}

Building on a mathematical correspondence with the incompressible Navier-Stokes equations, we have developed a numerical method for simulating the deformation of elastoplastic materials in the quasi-static limit that is analogous to the projection method in fluid mechanics [1]. The new method is most suitable for materials that can be well-described by the additive decomposition of the deformation rate into elastic and plastic parts. It is well-suited for a large class of materials (e.g. metals and amorphous solids such as metallic glasses), which typically undergo small elastic deformations and feature large elastic wave-speeds, making many plastic deformation problems intrinsically quasi-static. In such situations, the new method allows simulating realistic loading rates, which would be prohibitively computationally expensive using explicit methods.

The method is naturally implemented in an Eulerian framework. It is particularly well-suited to cases of straightforward boundary conditions, such as the simple shear experiments discussed in Section 4. We examined several basic features of shear band development in the STZ plasticity model, but the method could be adapted to look at a wide variety of problems in elastoplasticity, using STZ plasticity or other plasticity models. For example, detailed questions of shear band nucleation and growth, shear band interaction, or the role of structural inhomogeneities can be examined, and will be addressed elsewhere. Models with more complex physics, such as a coupling to real temperature evolving according to the diffusion equation, are also straightforward to incorporate. The derivation of the method should also be generalizable to the case of a non-constant and anisotropic stiffness tensor $\mathbf{C}$, and other objective stress rates, such as the Truesdell or Oldroyd stress rates.

As described in Section 5, the method can also be applied to problems involving moving free boundaries by using a suitable description of the boundary, such as the level set method. This framework may be well-suited to various fluid-structure interaction problems, offering some of the same advantages as the Eulerian hyperelasticity methods [27, 28, 26, 30, 32]. It may be interesting to examine the case of a quasi-static elastoplastic material interacting with an incompressible fluid, which would require a double projection to enforce both fluid incompressiblity and solid quasistaticity. As demonstrated in the final example in Subsec. 5.5, the method can also be intermixed with explicit timestepping, making it possible to simulate phenomena on multiple timescales.

The method presented here is underpinned by a close mathematical connection between the variables $(p, \mathbf{v})$ in the incompressible Navier-Stokes equations and $(\mathbf{v}, \boldsymbol{\sigma})$ in quasi-static elastoplasticity. This connection may therefore allow mathematical results for fluid mechanics to be translated to elastoplasticity. The incompressible limit of fluid mechanics has been extensively analyzed, often by examining the limit of small Mach number $M$, describing the ratio of a typical velocity to the sound speed, and playing a similar role to the artificial compressibility parameter [59]. Klainerman and Majda established that the solutions of the incompressible Navier-Stokes equations will match 

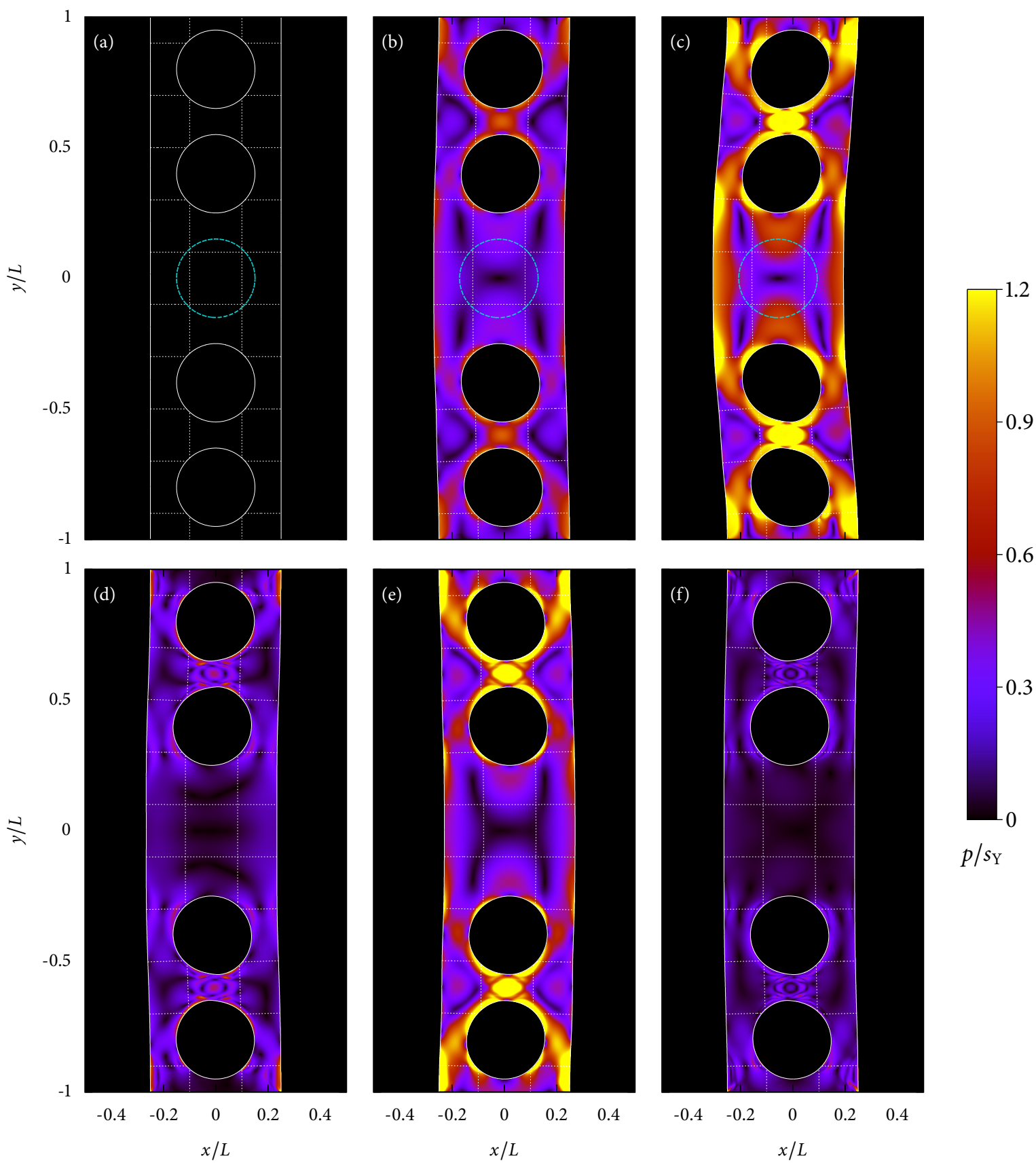

Figure 19: Plots of deviatoric stress $\left|\boldsymbol{\sigma}_{0}\right|$ for the load-release simulation. The top three snapshots are of the loading process simulated with the quasi-static method, at times of (a) $t=0$, (b) $t=2.5 \times 10^{5} t_{s}$, and (c) $t=t_{\mathrm{R}}=5 \times 10^{5} t_{s}$. The bottom three snapshots show the dynamics of the bar after the load is instaneously removed, simulated with the explicit method, at times of (d) $t=t_{\mathrm{R}}+2.5 t_{s}$, (e) $t=t_{\mathrm{R}}+5 t_{s}$, and (f) $t=t_{\mathrm{R}}+7.5 t_{s}$. The boundary of the bar is shown as the solid white line obtained as the zero contour of level set function $\phi$. The thin dashed white lines are the contours of the components of the reference map $\xi$ and show how the material is deformed. For plots (a) to (c), the dashed cyan circle shows the region where the bar is loaded. 

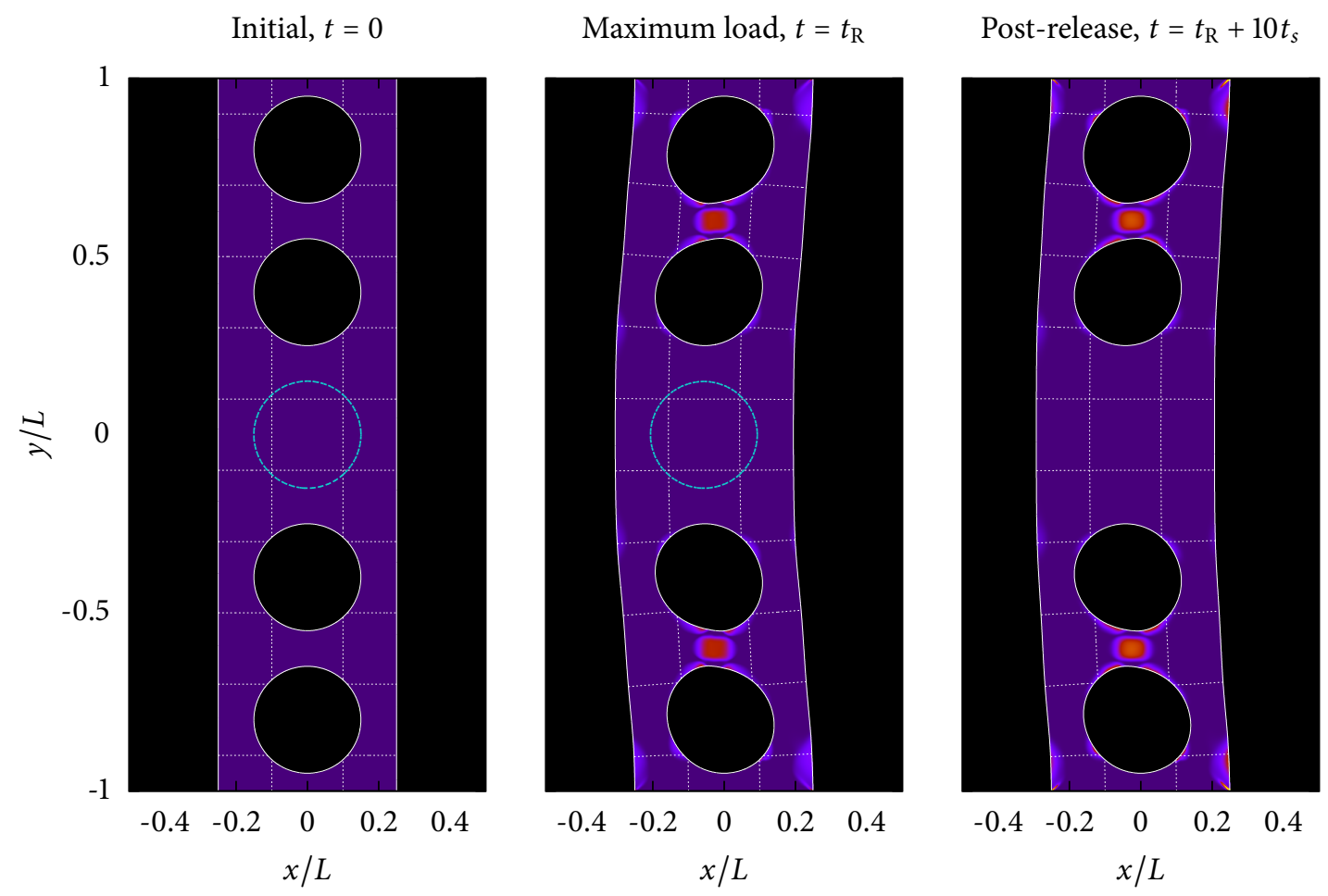

Figure 20: Plots of the effective temperature $\chi$ for the load-release simulation at three time points. The boundary of the bar is shown as the solid white line obtained as the zero contour of level set function $\phi$. The thin dashed white lines are the contours of the components of the reference map $\xi$ and show how the material is deformed. For plots (a) and (b), the dashed cyan circle shows the region where the bar is loaded. The color gradient for the effective temperature is the same as that used in Fig. 3.

the compressible Navier-Stokes equations in the limit of small $M$ [70]. In the context of turbulent combustion, where the Navier-Stokes equations are coupled to a reaction-diffusion equation, the zero Mach number limit has been examined by introducing perturbative expansions of the fields in powers of $M[71,72]$. These mathematical approaches provide possible avenues to establish rigorously that, in the long timescale limit, solutions to the full elastoplastic system will match the elastoplastic system with the quasi-staticity constraint.

A variety of advanced numerical approaches based on the fluid projection method have been developed, and it may be possible to translate these to elastoplasticity. Currently, the projection step that we employ is first-order accurate, but it is likely that high-order fluid projection methods [73, $74,75,76]$ could be adapted to the elastoplastic framework. The fluid projection step has also been implemented using finite elements within a finite-difference calculation $[77,25]$, which has the advantage of simplifying boundary conditions, and may provide a simpler solution for quasi-static elastoplasticity than the extrapolation formulae introduced in Subsec. 5.3. The fluid projection method has also been implemented on adaptive resolution grids [78], and if this was applied to elastoplasticity, it would allow for the investigation of the detailed structure of the localized shear bands that are a common feature of plasticity models. All of these interesting possibilities and directions should be systematically explored in future investigations. 


\section{Acknowledgments}

The authors thank Prof. Alexandre J. Chorin (University of California, Berkeley), Prof. Ken Kamrin (Massachusetts Institute of Technology), and Prof. James. R. Rice (Harvard University) for useful discussions about this work. C. H. Rycroft was supported by the Director, Office of Science, Computational and Technology Research, U.S. Department of Energy under contract number DEAC02-05CH11231. E. Bouchbinder acknowledges support from the Minerva Foundation with funding from the Federal German Ministry for Education and Research, the Israel Science Foundation (Grant No. 712/12), the Harold Perlman Family Foundation, and the William Z. and Eda Bess Novick Young Scientist Fund.

\section{Author contributions}

C. H. Rycroft developed the mathematical connection to the fluid projection method, formulated and programmed the explicit and quasi-static simulations, analyzed the results, created the figures, and wrote the manuscript. Y. Sui carried out initial tests of the explicit simulation method in the shear banding geometry of Section 4 . E. Bouchbinder identified the problem of simulating hypoelastoplasticity in the quasi-static regime, identified the physical importance of simulating realistic loading rates, introduced the constitutive relations, and was involved in writing the manuscript.

\section{Appendix A. Uniqueness of solution to the algebraic problem in Eq. 18}

In the quasi-static projection method, it is necessary to solve the algebraic problem given in Eq. 18, which can be rewritten as

$$
\nabla \cdot \boldsymbol{\sigma}_{*}=-\Delta t \nabla \cdot\left(\mathbf{C}: \nabla \mathbf{v}_{n+1}\right)
$$

by taking into account the symmetries of $\mathbf{C}$. Suppose that this equation must be solved on a fixed domain $\Omega$ where Dirichlet conditions for velocity are prescribed on the boundary $\partial \Omega$. Suppose that a second solution $\mathbf{v}_{n+1}^{\prime}$ exists. Hence the function $\mathbf{w}=\mathbf{v}_{n+1}^{\prime}-\mathbf{v}_{n+1}$ satisfies

$$
0=\nabla \cdot(\mathbf{C}: \nabla \mathbf{w})
$$

in $\Omega$, and $\mathbf{w}=\mathbf{0}$ on $\partial \Omega$. Multiplying the right hand side of Eq. A.2 and integrating gives

$$
0=\int_{\Omega} \mathbf{w} \cdot(\nabla \cdot(\mathbf{C}: \nabla \mathbf{w})) d^{3} \mathbf{x}=\int_{\partial \Omega} \hat{\mathbf{n}} \cdot(\mathbf{w} \cdot(\mathbf{C}: \nabla \mathbf{w})) d S-\int_{\Omega}(\nabla \mathbf{w}):(\mathbf{C}:(\nabla \mathbf{w})) d^{3} \mathbf{x} .
$$

The boundary integral will vanish since $\mathbf{w}=\mathbf{0}$ there, and hence

$$
0=\int_{\Omega}(\nabla \mathbf{w}): \mathbf{C}:(\nabla \mathbf{w}) d^{3} \mathbf{x}
$$

Since $\mathbf{C}$ is positive definite, it follows that $\nabla \mathbf{w}=\mathbf{0}$ and therefore $\mathbf{w}$ is a constant. Assuming $\partial \Omega \neq \varnothing$, the boundary condition will enforce that $\mathbf{w}=\mathbf{0}$, and hence that $\mathbf{v}_{n+1}^{\prime}=\mathbf{v}_{n+1}$ so that Eq. 18 has a unique solution. 
The above argument will also apply for traction-free boundary conditions discussed in Section 5. Equation 68 will lead to a Neumann-like condition $\mathbf{C}: \nabla \mathbf{w}=\mathbf{0}$, which will also lead to the boundary term in Eq. A.3 vanishing. In the case when only traction-free boundary conditions are applied, $\mathbf{w}$ will be a constant, so that $\mathbf{v}_{n+1}^{\prime}$ and $\mathbf{v}_{n+1}$ are equal up to a constant, which as discussed in Section 5 is physically reasonable.

\section{Appendix B. Adaptive sub-stepping}

As described in Subsec. 3.3, the plastic deformation $D^{\text {pl }}$ grows rapidly when $\bar{s}>s_{\mathrm{Y}}$, and this can cause the forward Euler timestepping procedure to lose accuracy, so that in a single timestep of size $\Delta t$, the change $\Delta \bar{s}$ in the deviatoric stress may be very large and significantly overshoot the yield surface. To solve this, an adaptive timestepping procedure is used that considers the coupled system of $\bar{s}$ and $\chi$ over the timestep $\Delta t$, in isolation from other terms. The procedure divides the interval $\Delta t$ into a number of substeps so that the change $\Delta \bar{s}$ at each substep remains within a fixed tolerance $\eta$; throughout this study, a value of $\eta=1 \%$ is used. To begin, the values of deviatoric stress and effective temperature at a given gridpoint are stored as $\bar{s}_{0}$ and $\chi_{0}$ respectively. The following algorithm is then used:

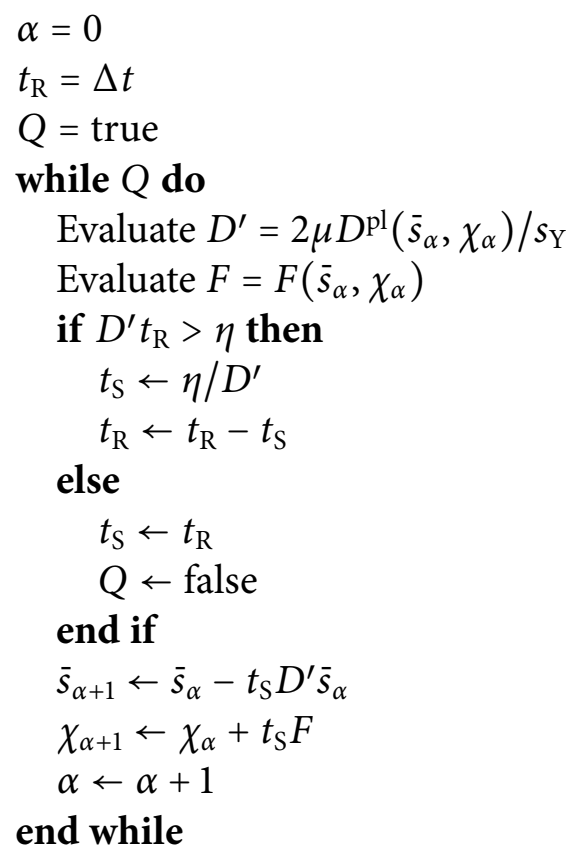

Here, a left arrow is used to signify a variable being updated. Within the algorithm, the variable $t_{\mathrm{R}}$ holds the remaining portion of the time interval to be considered. In the main loop, the algorithm determines whether the value of $\Delta \bar{s}$ for a timestep of size $t_{\mathrm{R}}$ is within the threshold $\eta$. If so, the algorithm takes a timestep of size $t_{\mathrm{R}}$ and terminates. Otherwise, it steps forward by the time interval $t_{\mathrm{S}}$ that makes $\Delta \bar{s}$ exactly match the threshold; it then subtracts $t_{\mathrm{S}}$ from $t_{\mathrm{R}}$ and repeats. Once the algorithm has terminated, corrected versions of plastic deformation and effective temperature change are evaluated according to

$$
\tilde{D}_{\mathrm{pl}}=\frac{\left(\bar{s}_{0}-\bar{s}_{\alpha}\right) s_{\mathrm{Y}}}{2 \mu \bar{s}_{0} \Delta t}, \quad \tilde{F}=\frac{\chi_{\alpha}-\chi_{0}}{\Delta t}
$$


These values are then used within the main finite-difference updates given in Eqs. 42 to 44 for the explicit simulation and Eqs. 50 to 52 for the quasi-static simulation. If $\eta$ is sufficiently large or $\Delta t$ is sufficiently small, so that the algorithm always terminates after a single step, then the main finite-difference updates reduce to the standard, fixed-timestep forward Euler procedure.

\section{References}

[1] A. J. Chorin, Numerical solution of the Navier-Stokes equations, Mathematics of Computation 22 (104) (1968) 745-762. doi :10 .1090/S0025-5718-1968-0242392-2.

[2] P. Lipinski, M. Berveiller, Elastoplasticity of micro-inhomogeneous metals at large strains, International Journal of Plasticity 5 (2) (1989) 149-172. doi : 10.1016/0749-6419 (89)90027-2.

[3] Y. Champion, C. Langlois, S. Guérin-Mailly, P. Langlois, J.-L. Bonnentien, M. J. Hÿtch, Nearperfect elastoplasticity in pure nanocrystalline copper, Science 300 (5617) (2003) 310-311. doi : 10.1126/science.1081042.

[4] D. L. Henann, K. Kamrin, A predictive, size-dependent continuum model for dense granular flows, Proceedings of the National Academy of Sciences 110 (17) (2013) 6730-6735. doi: 10.1073/pnas. 1219153110.

[5] M. Moner-Girona, A. Roig, E. Molins, E. Martínez, J. Esteve, Micromechanical properties of silica aerogels, Applied Physics Letters 75 (5) (1999) 653-655. doi : 10 . 1063/1. 124471.

[6] R. Vaidyanathan, M. Dao, G. Ravichandran, S. Suresh, Study of mechanical deformation in bulk metallic glass through instrumented indentation, Acta Materialia 49 (18) (2001) 3781-3789. doi : 10.1016/S1359-6454(01)00263-4.

[7] H. Xiao, O. Bruhns, A. Meyers, Elastoplasticity beyond small deformations, Acta Mechanica 182 (1-2) (2006) 31-111. doi : 10.1007/s00707-005-0282-7.

[8] J. Lubliner, Plasticity Theory, Dover, New York, 2008.

[9] M. E. Gurtin, E. Fried, L. Anand, The Mechanics and Thermodynamics of Continua, Cambridge University Press, Cambridge, 2010.

[10] E. Kröner, Allgemeine kontinuumstheorie der versetzungen und eigenspannungen, Archive for Rational Mechanics and Analysis 4 (1) (1959) 273-334. doi:10.1007/BF00281393.

[11] E. H. Lee, Elastic-plastic deformation at finite strains, Journal of Applied Mechanics 36 (1) (1969) 1-6. doi : 10.1115/1.3564580.

[12] L. Anand, C. Su, A theory for amorphous viscoplastic materials undergoing finite deformations, with application to metallic glasses, Journal of the Mechanics and Physics of Solids 53 (6) (2005) 1362-1396. doi :10.1016/j.jmps .2004.12.006. 
[13] C. Su, L. Anand, Plane strain indentation of a zr-based metallic glass: Experiments and numerical simulation, Acta Materialia 54 (1) (2006) 179 - 189. doi :10.1016/j . actamat .2005.08.040.

[14] P. Thamburaja, R. Ekambaram, Coupled thermo-mechanical modelling of bulk-metallic glasses: Theory, finite-element simulations and experimental verification, Journal of the Mechanics and Physics of Solids 55 (6) (2007) 1236-1273. doi:10.1016/j . jmps .2006.11.008.

[15] C. Reina, S. Conti, Kinematic description of crystal plasticity in the finite kinematic framework: A micromechanical understanding of $\mathbf{F}=\mathbf{F}_{e} \mathbf{F}_{p}$, Journal of the Mechanics and Physics of Solids 67 (2014) 40-61. doi:10.1016/j.jmps .2014.01.014.

[16] C. Truesdell, Hypo-elasticity, Indiana Univ. Math. J. 4 (1955) 83-133.

[17] R. Hill, A general theory of uniqueness and stability in elastic-plastic solids, Journal of the Mechanics and Physics of Solids 6 (3) (1958) 236-249. doi : 10.1016/0022-5096 (58)90029-2.

[18] W. Prager, An elementary discussion of definitions of stress rate, Quart. Appl. Math. 18 (1960) 403-407.

[19] J. C. Nagtegaal, J. E. De Jong, Some computational aspects of elastic-plastic large strain analysis, International Journal for Numerical Methods in Engineering 17 (1) (1981) 15-41. doi : 10 . $1002 /$ nme. 1620170103.

[20] J. Dienes, On the analysis of rotation and stress rate in deforming bodies, Acta Mechanica 32 (4) (1979) 217-232. doi : 10.1007/BF01379008.

[21] M. Kojić, K.-J. Bathe, Studies of finite element procedures-stress solution of a closed elastic strain path with stretching and shearing using the updated lagrangian jaumann formulation, Computers \& Structures 26 (1-2) (1987) 175-179. doi : 10 .1016/0045-7949 (87)90247-1.

[22] A. L. Eterovic, K.-J. Bathe, A hyperelastic-based large strain elasto-plastic constitutive formulation with combined isotropic-kinematic hardening using the logarithmic stress and strain measures, International Journal for Numerical Methods in Engineering 30 (6) (1990) 1099-1114. doi:10.1002/nme.1620300602.

[23] T. J. R. Hughes, J. Winget, Finite rotation effects in numerical integration of rate constitutive equations arising in large-deformation analysis, International Journal for Numerical Methods in Engineering 15 (12) (1980) 1862-1867. doi : 10.1002/nme.1620151210.

[24] J. C. Tannehill, Computational Fluid Mechanics and Heat Transfer, Taylor and Francis, 1997.

[25] J.-D. Yu, S. Sakai, J. A. Sethian, A coupled level set projection method applied to ink jet simulation, Interfaces and Free Boundaries 5 (4) (2003) 459-482.

[26] C. Liu, N. J. Walkington, An Eulerian description of fluids containing visco-elastic particles, Archive for Rational Mechanics and Analysis 159 (3) (2001) 229-252. doi:10.1007/ s002050100158. 
[27] B. J. Plohr, D. H. Sharp, A conservative Eulerian formulation of the equations for elastic flow, Advances in Applied Mathematics 9 (4) (1988) 481 - 499. doi:10.1016/0196-8858(88) 90025-5.

[28] J. A. Trangenstein, P. Colella, A higher-order Godunov method for modeling finite deformation in elastic-plastic solids, Communications on Pure and Applied Mathematics 44 (1) (1991) 41-100. doi:10.1002/cpa.3160440103.

[29] K. Kamrin, Stochastic and deterministic models for dense granular flow, Ph.D. thesis (2008).

[30] G.-H. Cottet, E. Maitre, T. Milcent, Eulerian formulation and level set models for incompressible fluid-structure interaction, ESAIM: Mathematical Modelling and Numerical Analysis 42 (2008) 471-492. doi : 10.1051/m2an:2008013.

[31] E. Maitre, T. Milcent, G.-H. Cottet, A. Raoult, Y. Usson, Applications of level set methods in computational biophysics, Mathematical and Computer Modelling 49 (11-12) (2009) 2161-2169. doi :10.1016/j.mcm.2008.07.026.

[32] K. Kamrin, C. H. Rycroft, J.-C. Nave, Reference map technique for finite-strain elasticity and fluid-solid interaction, Journal of the Mechanics and Physics of Solids 60 (11) (2012) 1952-1969. doi:10.1016/j.jmps.2012.06.003.

[33] B. Chen, J. J. Lu, C. H. Yang, J. H. Yang, J. Zhou, Y. M. Chen, Z. Suo, Highly stretchable and transparent ionogels as nonvolatile conductors for dielectric elastomer transducers, ACS Applied Materials \& Interfaces 6 (10) (2014) 7840-7845. doi:10.1021/am501130t.

[34] O. C. Zienkiewicz, P. N. Godbole, Flow of plastic and visco-plastic solids with special reference to extrusion and forming processes, International Journal for Numerical Methods in Engineering 8 (1) (1974) 1-16. doi : 10.1002/nme. 1620080102.

[35] L. B. Tran, H. S. Udaykumar, A particle-level set-based sharp interface cartesian grid method for impact, penetration, and void collapse, Journal of Computational Physics 193 (2) (2004) 4690-510. doi:10.1016/j.jcp.2003.07.023.

[36] C. H. Rycroft, F. Gibou, Simulations of a stretching bar using a plasticity model from the shear transformation zone theory, Journal of Computational Physics 231 (5) (2012) 2155-2179. doi:10.1016/j.jcp.2011.10.009.

[37] H. Hibbitt, P. Marcal, J. Rice, A finite element formulation for problems of large strain and large displacement, International Journal of Solids and Structures 6 (8) (1970) 1069-1086. doi:10.1016/0020-7683(70)90048-X.

[38] R. McMeeking, J. Rice, Finite-element formulations for problems of large elastic-plastic deformation, International Journal of Solids and Structures 11 (5) (1975) 601-616. doi: $10.1016 / 0020-7683(75) 90033-5$. 
[39] A. Needleman, On finite element formulations for large elastic-plastic deformations, Computers \& Structures 20 (1-3) (1985) 247-257. doi : 10 .1016/0045-7949 (85) 90074-4.

[40] J. Ponthot, Unified stress update algorithms for the numerical simulation of large deformation elasto-plastic and elasto-viscoplastic processes, International Journal of Plasticity 18 (1) (2002) 91-126. doi : 10.1016/S0749-6419(00)00097-8.

[41] M. L. Wilkins, Calculation of elastic-plastic flow, Tech. Rep. UCRL-7322, Lawrence Radiation Laboratory (1963).

[42] M. Ortiz, P. M. Pinsky, R. L. Taylor, Operator split methods for the numerical solution of the elastoplastic dynamic problem, Computer Methods in Applied Mechanics and Engineering 39 (2) (1983) 137-157. doi : 10 .1016/0045-7825 (83)90018-X.

[43] M. Ortiz, E. P. Popov, Accuracy and stability of integration algorithms for elastoplastic constitutive relations, International Journal for Numerical Methods in Engineering 21 (9) (1985) 1561-1576. doi : 10.1002/nme.1620210902.

[44] J. C. Simo, R. L. Taylor, A return mapping algorithm for plane stress elastoplasticity, International Journal for Numerical Methods in Engineering 22 (3) (1986) 649-670. doi:10.1002/nme. 1620220310.

[45] O. Bruhns, U. Rott, A viscoplastic model with a smooth transition to describe rateindependent plasticity, International Journal of Plasticity 10 (4) (1994) 347-362. doi : 10.1016/ 0749-6419 (94) 90037-X.

[46] J. C. Simo, T. J. R. Hughes, Computational inelasticity, Springer, New York, 1998.

[47] M. L. Falk, J. S. Langer, Dynamics of viscoplastic deformation in amorphous solids, Phys. Rev. E 57 (6) (1998) 7192-7205. doi:10.1103/PhysRevE.57.7192.

[48] E. Bouchbinder, J. S. Langer, I. Procaccia, Athermal shear-transformation-zone theory of amorphous plastic deformation. I. Basic principles, Phys. Rev. E 75 (3) (2007) 036107. doi: 10.1103/PhysRevE. 75.036107.

[49] E. Bouchbinder, J. S. Langer, Nonequilibrium thermodynamics of driven amorphous materials. I. Internal degrees of freedom and volume deformation, Phys. Rev. E 80 (3) (2009) 031131. doi:10.1103/PhysRevE.80.031131.

[50] E. Bouchbinder, T.-S. Lo, I. Procaccia, E. Shtilerman, Stability of an expanding circular cavity and the failure of amorphous solids, Phys. Rev. E 78 (2) (2008) 026124. doi : 10.1103/PhysRevE. 78.026124.

[51] F. Spaepen, A microscopic mechanism for steady state inhomogeneous flow in metallic glasses, Acta Metallurgica 25 (4) (1977) 407-415. doi :10.1016/0001-6160 (77)90232-2. 
[52] R. Huang, Z. Suo, J. Prevost, W. Nix, Inhomogeneous deformation in metallic glasses, Journal of the Mechanics and Physics of Solids 50 (5) (2002) 1011-1027. doi : 10.1016/S0022-5096(01) 00115-6.

[53] Y. Gao, B. Yang, T. Nieh, Thermomechanical instability analysis of inhomogeneous deformation in amorphous alloys, Acta Materialia 55 (7) (2007) 2319-2327. doi:10.1016/j . actamat. 2006.11 .027$.

[54] S. Osher, J. A. Sethian, Fronts propagating with curvature-dependent speed: Algorithms based on Hamilton-Jacobi formulations, Journal of Computational Physics 79 (1) (1988) 12-49. doi : 10.1016/0021-9991 (88)90002-2.

[55] J. A. Sethian, Level Set Methods and Fast Marching Methods, Cambridge University Press, 1996.

[56] S. J. Osher, R. P. Fedkiw, Level Set Methods and Dynamic Implicit Surfaces, Springer-Verlag, New York, 2003.

[57] C. H. Rycroft, E. Bouchbinder, Fracture toughness of metallic glasses: Annealing-induced embrittlement, Phys. Rev. Lett. 109 (2012) 194301. doi:10.1103/PhysRevLett.109.194301.

[58] Z. P. Bažant, A correlation study of formulations of incremental deformation and stability of continuous bodies, Journal of Applied Mechanics 38 (4) (1971) 919-928. doi:10.1115/1. 3408976.

[59] A. J. Chorin, A numerical method for solving incompressible viscous flow problems, Journal of Computational Physics 2 (1) (1967) 12-26. doi : 10 . 1016/0021-9991(67)90037-X.

[60] A. J. Chorin, J. E. Marsden, A Mathematical Introduction to Fluid Mechanics, Springer-Verlag, 1979.

[61] W. Slaughter, The Linearized Theory of Elasticity, Birkhäuser Boston, 2002.

[62] J. S. Langer, Shear-transformation-zone theory of plastic deformation near the glass transition, Phys. Rev. E 77 (2) (2008) 021502. doi:10.1103/PhysRevE.77.021502.

[63] E. Bouchbinder, J. S. Langer, Nonequilibrium thermodynamics of driven amorphous materials. II. Effective-temperature theory, Phys. Rev. E 80 (3) (2009) 031132. doi:10.1103/PhysRevE. 80.031132 .

[64] M. L. Manning, J. S. Langer, J. M. Carlson, Strain localization in a shear transformation zone model for amorphous solids, Phys. Rev. E 76 (5) (2007) 056106. doi:10.1103/PhysRevE. 76. 056106.

[65] M. L. Manning, E. G. Daub, J. S. Langer, J. M. Carlson, Rate-dependent shear bands in a shear-transformation-zone model of amorphous solids, Phys. Rev. E 79 (1) (2009) 016110. doi: 10.1103/PhysRevE.79.016110. 
[66] C.-W. Shu, S. Osher, Efficient implementation of essentially non-oscillatory shock-capturing schemes, J. Comp. Phys. 77 (2) (1988) 439-471. doi : 10.1016/0021-9991 (88) 90177-5.

[67] M. Sussman, P. Smereka, S. J. Osher, A level set method for computing solutions to incompressible two-phase flow, J. Comp. Phys. 114 (1) (1994) 146-159. doi :10.1006/jcph.1994.1155.

[68] T. D. Aslam, A partial differential equation approach to multidimensional extrapolation, J. Comp. Phys. 193 (1) (2004) 349-355. doi:10.1016/j . jcp.2003.08.001.

[69] D. L. Chopp, Some improvements of the fast marching method, SIAM Journal on Scientific Computing 23 (1) (2001) 230-244. doi :10.1137/S106482750037617X.

[70] S. Klainerman, A. Majda, Compressible and incompressible fluids, Communications on Pure and Applied Mathematics 35 (5) (1982) 629-651. doi :10.1002/cpa. 3160350503.

[71] A. Majda, J. A. Sethian, The derivation and numerical solution of the equations for zero Mach number combustion, Combustion Science and Technology 42 (3-4) (1985) 185-205. doi: 10.1080/00102208508960376.

[72] P. Embid, Well-posedness of the nonlinear equations for zero Mach number combustion, Communications in Partial Differential Equations 12 (11) (1987) 1227-1283. doi:10.1080/ 03605308708820526.

[73] J. B. Bell, P. Colella, H. M. Glaz, A second-order projection method for the incompressible Navier-Stokes equations, Journal of Computational Physics 85 (2) (1989) 257-283. doi : 10 . 1016/0021-9991 (89)90151-4.

[74] J. B. Bell, D. L. Marcus, A second-order projection method for variable-density flows, Journal of Computational Physics 101 (2) (1992) 334-348. doi : 10.1016/0021-9991 (92)90011-M.

[75] E. G. Puckett, A. S. Almgren, J. B. Bell, D. L. Marcus, W. J. Rider, A high-order projection method for tracking fluid interfaces in variable density incompressible flows, Journal of Computational Physics 130 (2) (1997) 269-282. doi:10.1006/jcph.1996.5590.

[76] D. L. Brown, R. Cortez, M. L. Minion, Accurate projection methods for the incompressible Navier-Stokes equations, Journal of Computational Physics 168 (2) (2001) 464-499. doi: $10.1006 / \mathrm{jcph} .2001 .6715$.

[77] A. Almgren, J. Bell, W. Szymczak, A numerical method for the incompressible Navier-Stokes equations based on an approximate projection, SIAM Journal on Scientific Computing 17 (2) (1996) 358-369. doi : 10.1137/S1064827593244213.

[78] C. Min, F. Gibou, A second order accurate projection method for the incompressible NavierStokes equations on non-graded adaptive grids, Journal of Computational Physics 219 (2) (2006) 912-929. doi:10.1016/j.jcp.2006.07.019. 